\title{
Migration und Bildungsarmut: Übertrittsrisiken im Schweizer Bildungssystem
}

\author{
Michael Beck und Franziska Jäpel
}

\section{Zusammenfassung}

Der Artikel untersucht Bildungsarmut im Schweizer Schulsystem dahingehend, ob sich auftretende Unterschiede nach Migrationshintergrund bei Übertritten im Sinne von Chancengerechtigkeit durch Schulleistungen erklären lassen oder ob Residualeffekte im Sinne allfälliger Diskriminierung auftreten. Während beim Übergang Primar-Sekundarschule keine Effekte erkennbar sind, zeigen sich solche beim (Nicht-) Übertritt in die nachobligatorische Ausbildung zu Ungunsten südosteuropäischer Herkunftsgruppen.

\section{Stichwörter}

Migration, Bildungsübertritt, Zertifikatsarmut, Diskriminierung

\section{$1 \quad$ Einleitung ${ }^{1}$}

Bildungsprozesse und der damit einhergehende Erwerb von Bildungszertifikaten spielen in Bezug auf die Integration von Personen mit Migrationshintergrund in die jeweilige Aufnahmegesellschaft, insbesondere in Bezug auf die Sozialintegration, eine zentrale Rolle (Esser 2006; Lockwood 1964). Somit ist Bildungsarmut von Personen mit Migrationshintergrund vor allem in klassischen Einwanderungsländern wie der Schweiz (Hoffmann-Nowotny 1973; Piguet 2006) eine gesellschaftliche Herausforde-

1 Dieser Beitrag baut in Teilen auf dem Artikel „Determinanten des Bildungserfolgs von Migranten“ (Beck et al. 2010) aus dem Sammelband „Bildungsverlierer - neue Ungleichheiten“ von Quenzel und Hurrelmann auf. Die sich daraus ergebenden Überschneidungen an verschiedenen Stellen sind der Lesbarkeit halber nicht jeweils separat ausgewiesen.

Diese Publikation wurde durch den Open Access Publikationsfonds der Pädagogischen Hochschule St.Gallen (PHSG) gefördert.

Die Originalversion dieses Kapitels wurde revidiert. Ein Erratum ist verfügbar unter https://doi.org/10.1007/978-3-658-19573-1_36 
rung. Wenn man davon ausgeht, dass Status in modernen Gesellschaften zu einem großen Teil über den Beruf und den mit diesem einhergehenden Privilegien vermittelt wird, so stellt der Erwerb von berufsrelevanten Bildungszertifikaten in Ländern mit einem Bildungssystem mit hoher beruflicher Spezifität (Allmendinger 1989) und einer starken Verknüpfung des Zugangs zu beruflichen Positionen über Bildung eine, wenn nicht sogar die zentrale Determinante von Status und den damit verbundenen Lebenschancen dar. Damit gesellschaftliche Integration und die Akzeptanz grundlegender Normen gelingen kann, ist eine faire Verteilung von Bildungszertifikaten im Sinne der Chancengerechtigkeit ${ }^{2}$ zentral. Dass jedoch Bildungsungleichheiten nach sozialer Herkunft und Geschlecht weiterhin bestehen, ist für die Schweiz bereits hinreichend belegt (Becker und Zangger 2013; Combet 2013; Hadjar und Berger 2010; Jann und Combet 2012; Moser et al. 2008; Moser et al. 1997; Neuenschwander 2012; Ramseier und Brühwiler 2003; Stadelmann-Steffen 2011). Hingegen konnte die Frage danach, ob der Migrationshintergrund in Bezug auf den Bildungserwerb einen Spezialfall der sozialen Herkunft darstellt (Kalter 2008) oder ob insbesondere beim Eintritt in die Berufsbildung systematische Ungleichheiten bestehen, welche möglicherweise ausschließlich auf den Migrationsstatus zurückgeführt und als Diskriminierung gewertet werden könnten, auf Basis der bisherigen Befundlage nicht abschließend geklärt werden.

Was den Besitz von Bildungszertifikaten betrifft, unterscheiden sich in der Schweiz Personen mit Migrationshintergrund - in Abhängigkeit ihres Generationenstatus - von der Bevölkerung ohne Migrationshintergrund: Hatten 201615 Prozent der über 15-Jährigen ohne Migrationshintergrund maximal einen obligatorischen Schulabschluss, so waren es bei der Bevölkerung mit Migrationshintergrund der ersten Generation mit 29 Prozent nahezu doppelt so viele, mit einem Migrationshintergrund der zweiten Generation mit 22 Prozent um die sieben Prozentpunkte mehr (BFS - Bundesamt für Statistik 2017c). Da in der Schweiz das Fehlen eines nachobligatorischen Bildungsabschlusses die Chance erwerbstätig anstelle von erwerbslos zu sein um mehr als die Hälfte reduziert (BFS 2017a, eigene Berechnungen), stellt Zertifikatsarmut einen erheblichen Risikofaktor für die Erwerbschancen betroffener Personen dar.

Bildungsarmut im Sinne von Zertifikatsarmut (vergleiche hierzu Quenzel und Hurrelmann in diesem Band) ist insbesondere in Ländern mit einem Ausbildungssystem mit hoher beruflicher Spezifität problematisch. Sowohl die Humankapitaltheorie (Becker 1993) als auch die Signaling Theorie (Arrow 1973; Spence 1973) gehen davon aus, dass Bildungszertifikate die Chancen auf erstrebenswerte berufliche Po-

2 Auch wenn der Begriff der Chancengerechtigkeit höchst unterschiedlich erfasst und verstanden werden kann, soll hier im theoretischen Sinne in Anlehnung an Kappus (2015: 12) Chancengerechtigkeit als Synonym des Begriffes „Equity“ definiert werden, als „[...] ein Konzept, das sich gegen die Benachteiligung und Diskriminierung von Menschen auf Grund ihrer Zugehörigkeit zu verschiedenen sozialen Gruppen bzw. aufgrund verschiedener sozialer Kategorisierungen wie sozialer Herkunft, Gender und Nationalität u. a. einsetzt.“. 
sitionen in Bezug auf Einkommen, Arbeitsplatzsicherheit und Prestige erhöhen. Wo jedoch die Humankapitaltheorie eher davon ausgeht, dass Zertifikate die Kompetenzen von Personen weitestgehend exakt beschreiben, nimmt die Signaling Theorie explizit an, dass Zertifikate nur eine von mehreren möglichen Informationsquellen für Arbeitgeberinnen und Arbeitgeber darüber darstellen, ob eine Person mit höherer Wahrscheinlichkeit die für eine bestimmte Jobposition benötigten Kompetenzen innehat. Vereinfacht wird hier davon ausgegangen, dass einem humankapitaltheoretischen Ansatz entsprechend Zertifikatsarmut mit Kompetenzarmut gleichzustellen ist, während gemäß dem signaltheoretischem Ansatz Zertifikatsarmut nicht zwingend Kompetenzarmut bedeuten muss und umgekehrt der Besitz eines Zertifikates nicht unbedingt die entsprechenden Kompetenzen voraussetzt. Im Sinne der Chancengerechtigkeit bestünde dann ein Ungleichgewicht, wenn vergleichbare Kompetenzen und Potentiale nicht entsprechend zertifiziert werden, sondern der Erwerb dieser Zertifikate von anderen Faktoren, wie beispielsweise einem Migrationshintergrund abhängen.

Der Beitrag geht der Frage nach, ob und in welchem Ausmaß Personen mit Migrationshintergrund im Schweizer Bildungssystem Risiken ausgesetzt sind, die zu einer Zertifikatsarmut führen (können). Die nachfolgenden Ausführungen sind wie folgt gegliedert: Zunächst wird das Bildungssystem der Schweiz skizziert, sowie Befunde zur Bildungsbeteiligung von Schülerinnen und Schülern mit Migrationshintergrund zusammengefasst. Darauf aufbauend werden ausgesuchte theoretische Erklärungsmuster für eine allfällige Schlechterstellung von Personen mit Migrationshintergrund vorgestellt. Anschließend folgen Analysen zu zwei zentralen Übergangspunkten: von der Primarstufe auf die Sekundarstufe I sowie von der Sekundarstufe I in die nachobligatorische Ausbildung. Der Artikel endet mit einem Fazit.

\section{Migration und Bildungsarmut am Beispiel der Schweiz}

Weil Bildungschancen wesentlich von den Restriktionen und selektiven Anreizen des Bildungssystems abhängen und im Falle von Bevölkerungsgruppen mit Migrationshintergrund die Kenntnisse über Bildungswege im Aufnahmeland eine wichtige Rolle für die Bildungsteilhabe spielen, ist es notwendig, das Schweizer Bildungssystem zu beschreiben. Es lässt sich eine generelle Grundstruktur skizzieren, deren Charakteristika in vielen Punkten mit dem deutschen Bildungssystem vergleichbar sind.

\section{Struktur und Organisation des Schweizer Bildungssystems}

Das Bildungssystem der Schweiz ist, ähnlich wie das deutsche, in Stufen und Typen unterteilt, wobei die Hauptverantwortung für das Bildungswesen bei den 26 Kantonen liegt. Auf nationaler Ebene wird es durch die Schweizerische Konferenz der 
kantonalen Erziehungsdirektoren (EDK) koordiniert. Auf kantonaler Ebene wird das Schulwesen durch jeweils eigene Schulgesetze geregelt und die einzelnen Gemeinden verfügen über eine relativ hohe Autonomie in Bildungsangelegenheiten, was zu unterschiedlichen und den lokalen Gegebenheiten angepassten strukturellen Lösungen führen kann. Die Unterrichtssprache ist je nach Sprachregion Deutsch, Französisch, Italienisch oder Rätoromanisch. Gesamtschweizerisch einheitlich geregelt sind Schuleintrittsalter, Beginn und Dauer des Schuljahres sowie Dauer der obligatorischen Schulzeit (EDK 2017). Abbildung 1 gibt einen Überblick über den Aufbau des Schweizer Bildungssystems.

Die obligatorische Schulzeit umfasst neun Schuljahre (EDK 2017). Am Ende der Primarstufe (in der Regel nach der sechsten Klasse, in manchen Kantonen nach der vierten oder fünften Jahrgangsstufe) wird für die Schulkinder entschieden, welchen Sekundarschultyp sie während der dreijährigen Sekundarstufe I besuchen werden. Grundsätzlich gibt es zwei Typen, die sich in ihren Leistungsniveaus unterscheiden: den Schultyp mit Grundanforderungen und den Schultyp mit erweiterten Anforderungen. In einigen Kantonen gibt es drei Schultypen: einen Schultyp mit Grundanforderungen, einen mit mittleren Anforderungen und einen mit höheren Anforderungen. Es sind jedoch auch Mischformen möglich, bei welchen eine Differenzierung nach Anforderungsniveau nur in bestimmten Fächern oder auch innerhalb einer Klasse stattfindet. Der Übertritt an Schulen mit Grundanforderungen erfolgt ohne Prüfung, derjenige an Schulen mit erweiterten Anforderungen stützt sich meist auf Empfehlungen der Primarlehrpersonen. Ausschlaggebend sind schulische Leistungen - insbesondere in der ersten Landessprache des jeweiligen Kantons, in Fremdsprachen und in Mathematik. Mit Abschluss der Sekundarstufe I endet die obligatorische Schulzeit. Der Übertritt in die stark gegliederte Sekundarstufe II mit ihrem vielfältigen Angebot an gymnasialer, mittlerer und beruflicher Bildung erfolgt je nach Eignung und Interesse der Schülerinnen und Schüler. Bereits in der Sekundarstufe I können geeignete Schülerinnen und Schüler in der neunten Klasse eine Klasse zur gymnasialen Vorbildung besuchen und nach zwölf Schuljahren mit einer gymnasialen Matura abschließen. Vor allem im Bereich der beruflichen Grundbildung können sie zwischen verschiedenen Ausbildungsvarianten (duale Berufslehre und Allgemeinbildung, vollzeitschulische Ausbildung) wählen und haben zudem die Option, zeitgleich oder im Anschluss an die Berufslehre die Berufsmaturität zu erlangen.

Insgesamt kennzeichnen das Schweizerische Bildungssystem eine vergleichsweise hohe Stratifizierung und Segmentierung mit einer frühen und ausgeprägten institutionellen Selektion beim Übertritt von der Primar- in die Sekundarstufe I (Beck 2015; Buchmann und Dalton 2002) sowie einem breiten berufsbildenden Angebot, dem ein relative geschlossenes Hochschulsystem gegenübersteht (Griga 2014; Schumann 2011). Dieser strukturellen Organisation wird, ähnlich wie im deutschen Bildungssystem, eine Ablenkungswirkung nachgewiesen, die dazu führt, dass sozial schlechter gestellte Jugendliche sich eher in die berufsbildenden Ausbildungen ,ablenken lassen (Jäpel 2017; Glauser 2015; Schindler 2012; Becker und Hecken 2008; Shavit und 
Abbildung 1 Struktur und Aufbau des Schweizer Bildungssystems

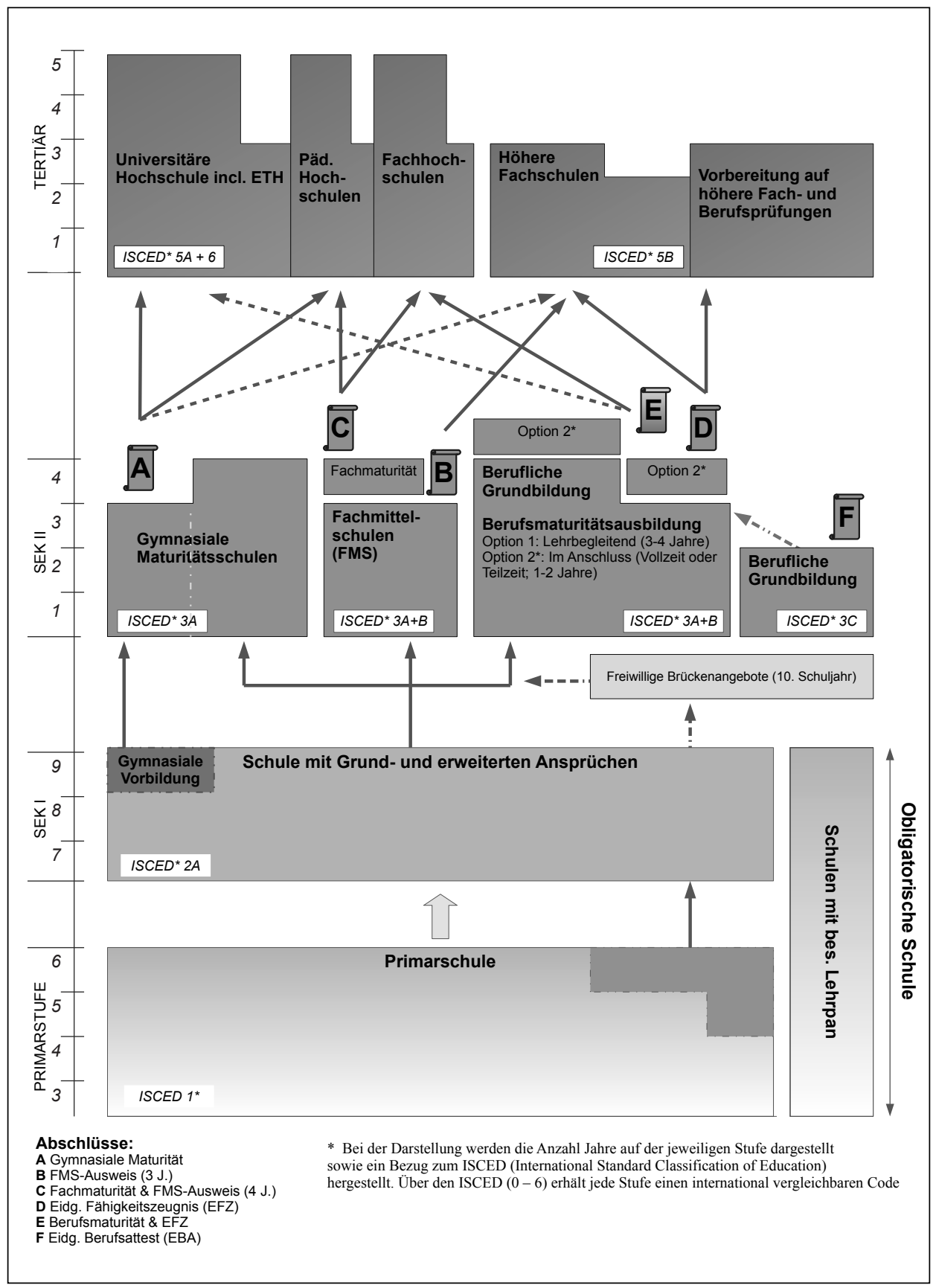

Quelle: EDK (2017), eigene Darstellung 
Muller 2000). Die Übergänge zwischen den verschiedenen Ausbildungsstufen sind jedoch zeitlich unterschiedlich gelagert. Wie für Deutschland bereits mehrfach belegt (Schindler 2012; Kristen und Dollmann 2010), stellen auch im Bildungssystem der Schweiz diese Übergänge entscheidende selektive Hürden dar (Becker et al. 2013; Kronig 2007; Kronig et al. 2000; Moser und Rhyn 2000) an denen maßgebliche Entscheidungen für die weitere Bildungslaufbahn von Kindern und Jugendlichen und deren Bildungschancen getroffen werden.

\section{Zur Situation von Personen mit Migrationshintergrund im Schweizer Bildungssystem}

Fragen bezüglich des Bildungszugangs verschiedener Personengruppen, migrationsbedingter Disparitäten im Bildungserfolg und nach Übergangsmustern im Bildungssystem werden nicht erst seit der Diskussion um die PISA-Ergebnisse von verschiedenen wissenschaftlichen Disziplinen untersucht (zum Beispiel Jäpel 2017; Tjaden und Scharenberg 2016; Beck 2015; Glauser 2015; SKBF 2014; Becker et al. 2013; Haeberlin et al. 2004b; Meyer et al. 2003).

Abbildung 2 Besuchte Schulform auf Sekundarstufe I nach Anspruchsniveau und Migrationshintergrund

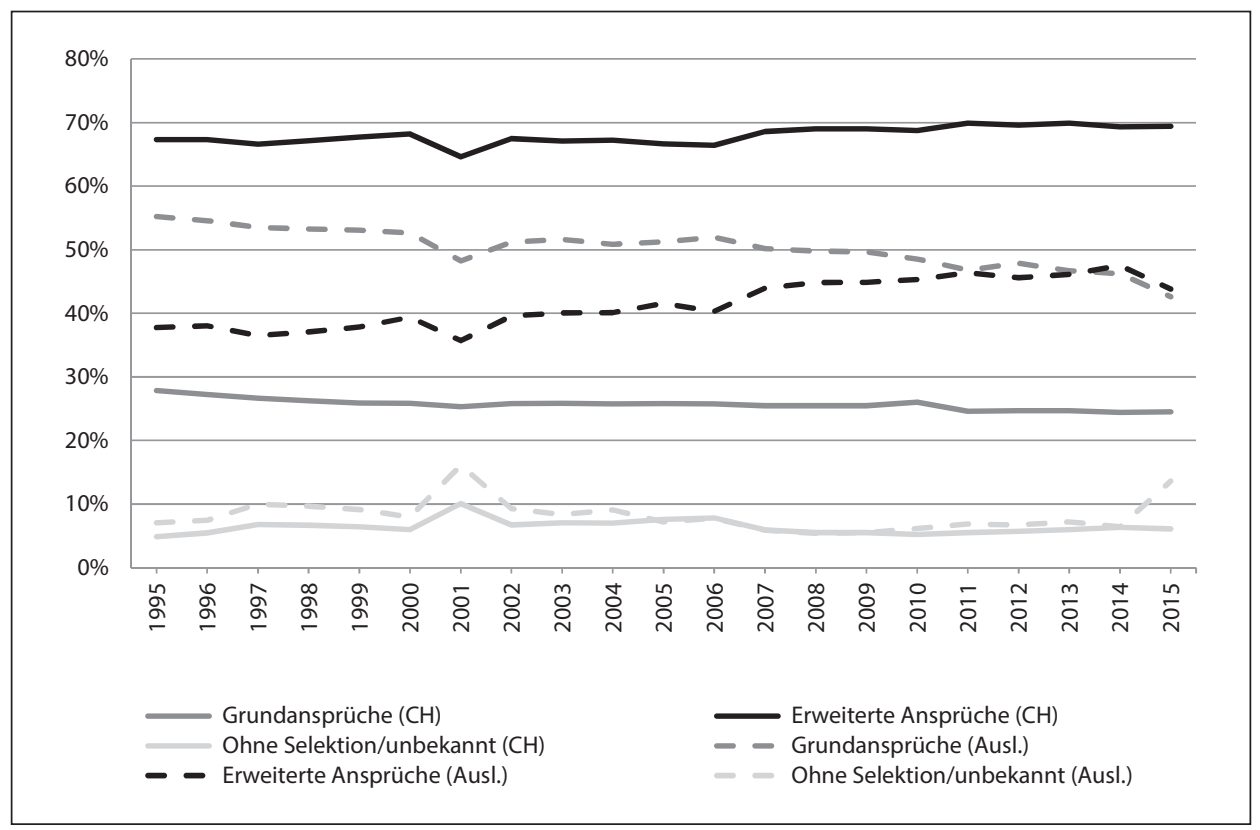

Quelle: BFS (2017b), eigene Darstellung 
Abbildung 3 Besuchte Schulform auf Sekundarstufe II nach Anspruchsniveau und Migrationshintergrund

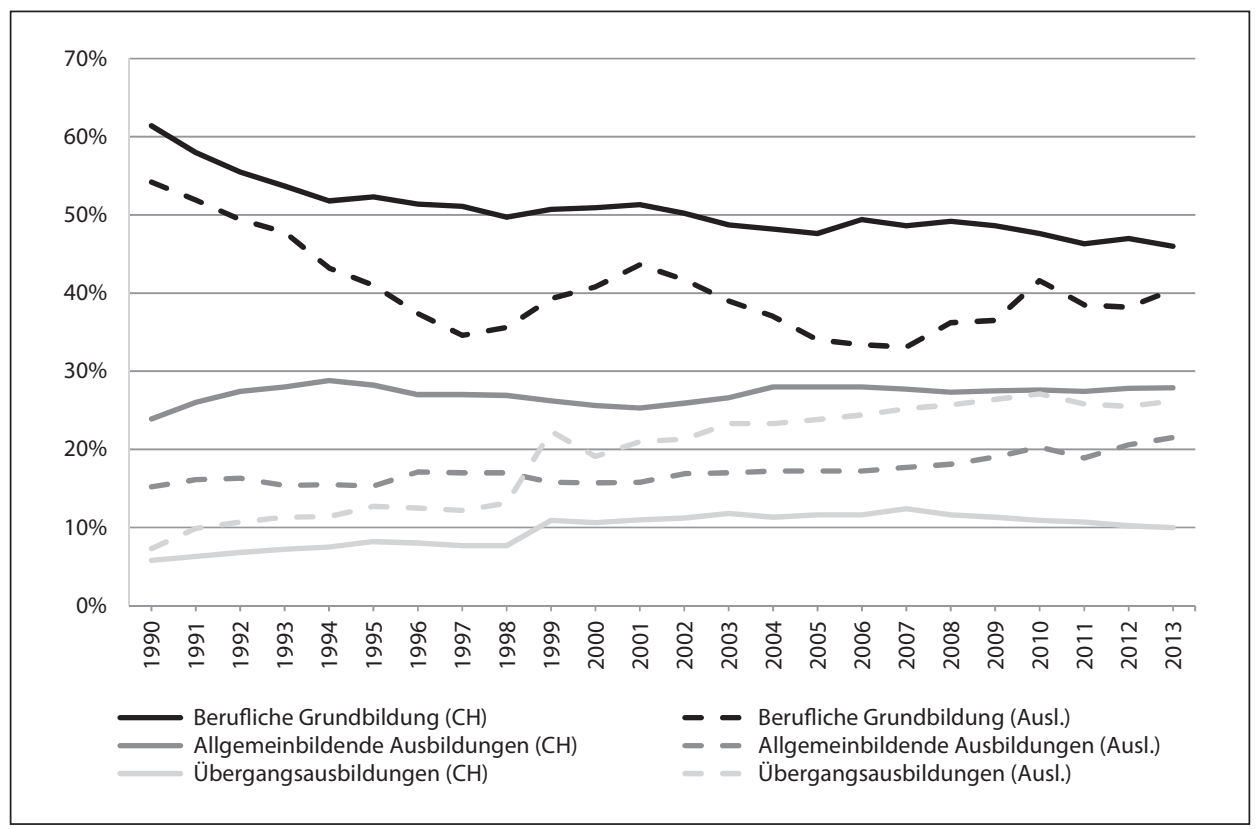

Quelle: BFS (2017d), eigene Darstellung

Im Schuljahr 2015/2016 betrug der Anteil ausländischer Schülerinnen und Schüler in der obligatorischen Schule in der Schweiz rund 27 Prozent, was gegenüber 1999/2000 einen Anstieg von rund vier Prozent bedeutet (BFS 2017b). Wie in Abbildung 2 ersichtlich ist, waren Schulkinder ausländischer Nationalität ${ }^{3}$ bis ungefähr 2011 auf der Sekundarstufe I häufiger in Schulformen mit Grundanforderungen anzutreffen, als in solchen mit erweiterten Anforderungen. Erst 2011 ist für ausländische Schülerinnen und Schüler eine Angleichung der Anteile für beide Schulformen sichtbar, im Vergleich zu inländischen Schülerinnen und Schülern sind sie jedoch immer noch auf Schulformen mit Grundanforderungen überproportional häufig vertreten.

Beim Übergang von der Sekundarstufe I auf die Sekundarstufe II (Abbildung 3) zeigt sich, dass die Anteile der Übergänge in die berufliche Grundbildung sowohl für inländische, als auch für ausländische Schülerinnen und Schüler im Laufe der 9oer Jahre stark abgenommen haben (26 Prozent). Während insbesondere ausländische

3 Da differenzierte Angaben zum Migrationshintergrund beim Schweizerischen Bundesamt für Statistik erst ab dem 15. Lebensalter vorliegen, können Schülerinnen und Schüler mit Migrationshintergrund im obligatorischen Schulsystem nur (in unzureichender Weise) über die Nationalität identifiziert werden. 
Schülerinnen und Schüler im Zeitverlauf immer häufiger in allgemeinbildende Schulen eintreten, nimmt auch der Anteil unter ihnen, welche in eine Übergangsausbildung übertreten im Zeitverlauf zu.

Betrachtet man Personen, welche nicht unmittelbar in eine zertifizierende Ausbildung auf der Sekundarstufe II übergehen (Abbildung 4), zeigt sich im Verlaufe der 9oer Jahre ein massiver Anstieg des Risikos für ausländische Schülerinnen und Schüler, nicht direkt in zertifizierende Anschlusslösungen überzugehen. Im Verlaufe der Jahrtausendwende zeigt sich jedoch ein klarer Trendwechsel: 2013 verließen nur noch rund zwölf Prozent der ausländischen Schülerinnen und Schüler ohne direkte zertifizierende Anschlusslösung die Sekundarstufe I, bei inländischen Schülerinnen und Schülern waren es hingegen 16 Prozent.

Verschiedene Studien beschäftigen sich mit Fragen zu Bildungsdisparitäten in der Schweiz und den ihnen zugrundeliegenden Mechanismen beim Übergang von der Primarschule in die Sekundarstufe I (Beck 2015; Beck et al. 2010; Becker et al. 2013; Böni und Salm 2008; Trautwein und Baeriswyl 2007; Kronig 2007; Haeberlin et al. 2004a; Ramseier und Brühwiler 2003; Moser 2001; Moser und Rhyn 2000). Der folgende Überblick soll den Forschungsstand zu Bildungsübergängen in der Schweiz mit einem expliziten Fokus auf Zertifikatsarmut skizieren.

Bereits vor Schuleintritt gibt es Anhaltspunkte, dass Kinder mit Migrationshintergrund seltener vorschulische oder frühkindliche Bildungseinrichtungen besuchen, wobei hier keine einheitliche Erfassung vorliegt (zum Problem der Erfassung von Kindern in frühkindlichen Bildungseinrichtungen vergleiche Stamm et al. 2009). Zudem werden Schülerinnen und Schüler mit Migrationshintergrund häufiger in Einschulungsklassen überwiesen, zeigen ein höheres Risiko für Schulabbruch und haben im gesamten Verlauf der obligatorischen Schulkarriere ein höheres Risiko, nach besonderem Lehrplan unterrichtet zu werden (Kronig 2003; Kronig et al. 2000; SKBF 2007; 2010; Stamm et al. 2011). Es gibt Hinweise, dass die häufigere Überweisung in Sonderklassen nicht ausschließlich leistungsbasiert erfolgt (Kronig 2003).

Migrationshintergrund und Fremdsprachigkeit gehen tendenziell mit geringeren schulrelevanten Kompetenzen sowohl auf der Primar- als auch der Sekundarstufe einher, auch unter Kontrolle der sozialen Herkunft und anderer Faktoren wie Motivation und Geschlecht ${ }^{4}$. Dabei besteht bereits in der ersten Klasse ein Unterschied zu Ungunsten fremdsprachiger Schülerinnen und Schüler in Deutsch und Mathematik, welcher sich im Verlauf der Primarschule vergrößert (Moser et al. 2011; 2008). Die Nachteile können unter Kontrolle von Geschlecht, sozialer Herkunft und kognitiven Grundfähigkeiten sowohl im Lernstand als auch im Lernzuwachs überwiegend statistisch erklärt werden, dies gilt für Unterschiede nach sozialer Herkunft hingegen nicht.

4 Vergleiche für PISA: Konsortium Pisa.ch (2010); Moser (2002); Ramseier und Brühwiler (2003), für TIMMS: Moser et al. (1997), für die IEA-Reading-Literacy-Study: Rüesch (1998), für die Zürcher Lernstandserhebungen Moser et al. (2011); Moser et al. (2008); Moser und Rhyn (2000). 
Abbildung 4 Schülerinnen und Schüler ohne Anschlusslösung auf Sekundarstufe II nach Migrationshintergrund

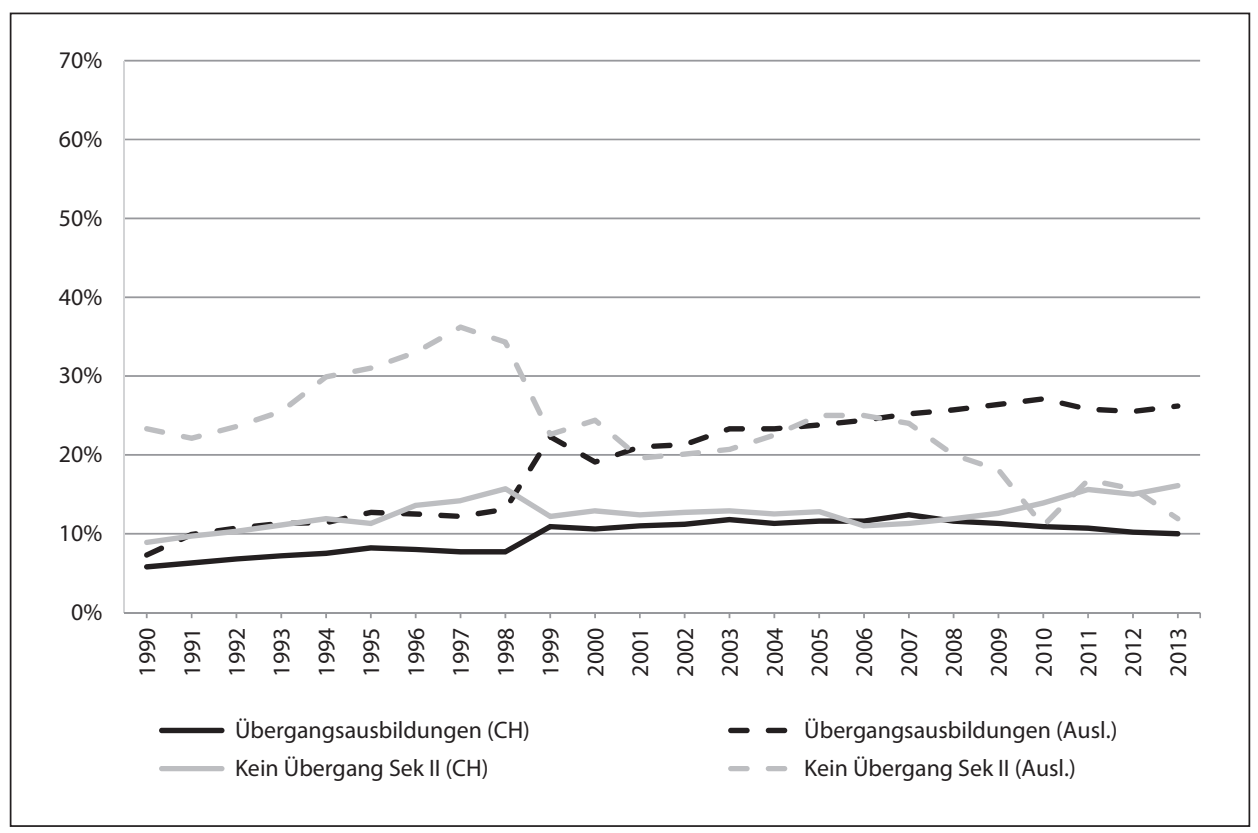

Quelle: BFS (2017d), eigene Darstellung

Die selektiven Mechanismen beim Eintritt in die Sekundarstufe I sind deshalb von Bedeutung, weil sie den weiteren Bildungsverlauf bis hin zum Erwerbseintritt wesentlich vorstrukturieren. Der Einfluss von Merkmalen der Herkunft hat hier weitreichende Folgen, da ein Scheitern bereits früh in der Bildungskarriere das Risiko für Bildungsarmut erhöht. Verschiedene Untersuchungen kommen zum Ergebnis, dass Noten und Leistungen zwar einen großen Teil der Übergangsmuster erklären, dass je nach Übergangsschwelle darüber hinaus jedoch unterschiedliche Risiken nach migrationsbedingter und sozialer Herkunft angenommen werden können (Beck et al. 2010; Becker et al. 2013; Meyer 2003b). Die amtliche Statistik sowie zahlreiche Studien die sich mit den Bildungschancen von Personen mit Migrationshintergrund beschäftigen, verweisen auf besondere Risiken im Hinblick auf Bildungszugang und Bildungserwerb. Da das Schweizer Bildungssystem mit seiner vergleichsweise hohen Stratifizierung bzw. frühem Tracking (Allmendinger 1989) viele Übergangspunkte aufweist, gilt ein Übertritt in einen Sekundarschultyp mit Grundanforderungen als erster Risikofaktor dafür, kein nachobligatorisches Bildungszertifikat zu erreichen. Jedoch ist die Befundlage zum Übertritt von Schülerinnen und Schülern mit Migrationshintergrund nicht ohne Widersprüche: Während Haeberlin et al. (2004b) und Kronig (2007) unterschiedliche Übertrittsquoten nach Geschlecht und Migrations- 
hintergrund (deutlich zum Nachteil von Schülern mit Migrationshintergrund) bei vergleichbarer Leistung zeigen können, so finden sich in anderen Studien (Beck 2015; Beck et al. 2010; Becker et al. 2013; Moser et al. 2017; Moser und Rhyn 2000; Ramseier und Brühwiler 2003; Trautwein und Baeriswyl 2007) keine bis leicht positive Effekte für einen Übertritt von Schülerinnen und Schülern mit Migrationshintergrund, wenn für soziale Herkunft und Schulleistungen kontrolliert wird. Die meisten Befunde sprechen zudem dafür, dass bei der Notengebung unter Kontrolle standardisierter, lehrpersonenunabhängiger Tests keine negativen Residualeffekte für Schülerinnen und Schüler mit Migrationshintergrund bestehen (Beck et al. 2010; Becker et al. 2013; Moser und Rhyn 2000; Ramseier und Brühwiler 2003; Trautwein und Baeriswyl 2007).

Bezüglich des Übertritts am Ende der Sekundarstufe I weißt die Befundlage auf Nachteile bestimmter Gruppen von Personen mit Migrationshintergrund hin. Die Daten der amtlichen Statistik für das Jahr 2013 (BFS 2017d) zeigen, dass ausländische Jugendliche mit 62 Prozent seltener als Schweizer Jugendliche (74 Prozent) direkt in eine zertifizierende Ausbildung der Sekundarstufe II eintreten und mehr als doppelt so häufig (26 Prozent) eine Übergangs- oder Zwischenlösung beginnen (Schweizer Jugendliche: zehn Prozent). Analysen mit Daten der TREE Befragung (TREE 2016) legen nahe, dass Schülerinnen und Schüler mit einem Migrationshintergrund aus südosteuropäischen Ländern (zweite Migrationswelle) auch unter Kontrolle der sozialen Herkunft und der Sekundar-I-Schulform häufiger keine nachobligatorische Ausbildung absolvieren (Glauser 2018; Meyer 2003a). Gleichzeitig kann gezeigt werden, dass Schülerinnen und Schüler mit Migrationshintergrund der ersten Generation auch dann häufiger von Ausbildungslosigkeit betroffen sind, wenn für Schultyp, Schulleistung und soziale Herkunftsmerkmale kontrolliert wird (Beck et al. 2010; Meyer 2003a). Schülerinnen und Schüler mit Migrationshintergrund, welche in der Schweiz geboren wurden, zeigen unter Kontrolle dieser Faktoren kein erhöhtes Risiko für Ausbildungslosigkeit (Beck et al. 2010; Hupka 2003). Jedoch ist ihr Risiko, nach der obligatorischen Schule in eine nichtzertifizierende Zwischenlösung überzugehen oder ganz ohne Anschlusslösung zu verbleiben, auch unter Kontrolle der genannten Faktoren erhöht (Glauser 2018; Meyer 2003a). Hinweise für Diskriminierung beim Bewerbungsprozess zeigen sich zudem bei Fibbi et al. (2006).

\section{Migrationshintergrund: Eine Frage der Definition}

Für viele Studien und in amtlichen Daten stehen oft nur begrenzte Angaben zur Staatsangehörigkeit oder der zu Hause gesprochenen Sprache zur Verfügung, welche lediglich eine relativ grobe Unterscheidung nach Migrationshintergrund erlauben. Um den Migrationshintergrund nach Diefenbach (2007) als Bewegungen von Personen oder Personengruppen, die einen dauerhaften Wohnortwechsel bedingen, verbunden mit einem durch Distanz hervorgerufenen kulturellen Aspekt, hinreichend 
abzubilden, ist es zunächst ausreichend, alleine das Vorliegen einer Migrationsgeschichte in der Familie zu identifizieren. Unter der Annahme, dass die (vor allem kulturellen) Ressourcen von Individuen ebenso wie deren Werthaltungen stark durch die Gesellschaftsstrukturen ihrer Herkunftsländer geprägt sind, kann dieser Heterogenität durch eine Dichotomisierung nach Merkmalen wie Inland/Ausland, fremdsprachig/deutschsprachig oder Migrationshintergrund ja/nein jedoch nicht hinreichend Rechnung getragen werden, zumindest dann nicht, wenn die durch Ressourcen und Werthaltungen direkt beeinflussten Unterschiede im Entscheidungsverhalten (und um solche handelt es sich bei Bildungsentscheidungen) adäquat erklärt werden sollen (für eine Diskussion und Beispiele des Effekts unterschiedlicher operationaler Definitionen sei auf Beck und Edelmann (2016); Gresch und Kristen (2011) sowie Kemper (2010) verwiesen).

Grundsätzlich zeigt sich in der aktuellen Forschung eine Kategorisierung nach dem Generationenstatus als modus opperandi der Operationalisierung von Migrationshintergrund. Der zentrale Aspekt dabei ist das Geburtsland. Als Personen mit Migrationshintergrund der ersten Generation werden solche Personen verstanden, welche in einem anderen Land geboren sind, also selbst Migrationserfahrungen gemacht haben. In der Schweiz geborene Personen, deren Eltern nicht in der Schweiz geboren wurde, werden normalerweise als Personen mit Migrationshintergrund der zweiten Generation bezeichnet, wobei es darüber hinaus eine große Anzahl unterschiedlicher Varianten (Olczyk et al. 2016) gibt. Um Unterschiede in den Migrationsmotiven und den Herkunftskulturen adäquat abzubilden, ist zudem die Berücksichtigung des Herkunftskontextes (meist über das Herkunftsland) notwendig. In der Schweizer Forschung wird häufig nach erster und zweiter Migrationswelle unterschieden: Während unter der ersten Migrationswelle Personen aus Italien und Spanien verstanden werden, werden unter Angehörigen der zweiten Migrationswelle überwiegend Personen aus Südosteuropa (Staaten des ehemaligen Jugoslawien, Albanien und Türkei), Portugal sowie teilweise Sri Lanka zusammengefasst (Beck 2015; Glauser 2015; Beck et al. 2010; Meyer 2003a).

\section{Theoretische Grundlagen allgemeiner und migrationsbedingter Bildungsungleichheiten}

Soziologische Handlungstheorien basieren meist (wenn auch nicht immer explizit) auf der Annahme, dass Personen Handlungen mit dem Ziel ausführen, ein möglichst hohes, subjektives psychisches und physisches Wohlbefinden zu erreichen (Jasso 2007; Lindenberg 1984). Die Maximierung des Wohlbefindens wird dabei über sogenannte Zwischengüter erreicht, von welchen dem Begriff Status eine zentrale Rolle zukommt. Da dieser in modernen Gesellschaft zu einem großen Teil über den Beruf vermittelt wird, spielt der Erwerb von hierfür notwendigen Bildungszertifikaten eine, wenn nicht sogar die zentrale Rolle. 
Bei der Erklärung von Bildungsungleichheiten wird häufig das theoretische Modell der strukturell-individualistischen Erklärung von Bildungsungleichheiten angewandt (Jäpel 2017; Beck 2015; Glauser 2015; Beck et al. 2010). Dieses geht davon aus, dass ein zentraler Mechanismus zur Entstehung sozialer aber auch migrationsbedingter Bildungsungleichheiten Bildungsentscheidungen an unterschiedlichen Punkten des Bildungssystems sind, welche durch subjektive Kosten-Nutzen-Evaluationen beeinflusst sind. Dabei sind sowohl die Kosten-Nutzen-Evaluationen als auch die Freiheit der Entscheidung selbst durch gesellschaftliche und institutionelle Rahmenbedingungen beeinflusst. Hier wird in Anlehnung an das durch Coleman (1990) geprägte Modell der soziologischen Tiefenerklärung, welches den Zusammenhang zwischen Variablen - wie dem Bildungssystem und den erreichten Bildungszertifikaten - durch individuelle Handlungsentscheidungen erklärt, zwischen den folgenden drei Ebenen unterschieden:

Makroebene: Hier sind gesellschaftliche Rahmenbedingungen und damit einhergehende rechtliche Aspekte wie die gesetzliche Schulpflicht und das Ausländerrecht sowie Aggregate, wie die soziale und migrationsbedingte Bildungs- und Arbeitsmarktbeteiligung, angesiedelt.

Mesoebene: Hier sind schulische Rahmenbedingungen wie die Ausstattung von Schulen, die Zusammensetzung des Lehrkörpers (zum Beispiel nach Geschlecht, Ausbildung, sozialer Herkunft, Migrationshintergrund), die Zusammensetzung nach unterschiedlichen Dimensionen von lernrelevanter Heterogenität (soziale Herkunft, Migrationshintergrund, Kompetenzniveau und -streuung sowie Fremdsprachigkeit) angesiedelt. Ebenso sind Merkmale der Lehrpersonen und die damit zusammenhängenden Praktiken der Kompetenzvermittlung, der Notenvergabe, der Übertrittsempfehlungen (am Ende der Primarstufe) sowie der Berufswahlunterstützung (auf der Sekundarstufe I) zu nennen.

Mikroebene: Hier sind individuelle Faktoren von Schülerinnen und Schülern wie deren Geschlecht, Migrationshintergrund sowie deren soziale Herkunft und die mit den letzteren einhergehenden individuellen und familiären Ressourcenausstattungen angesiedelt (Beck 2015; Becker und Lauterbach 2010; Bourdieu 1983). Diese beeinflussen, gemeinsam mit genetischen Voraussetzungen, den schulischen Kompetenzerwerb sowie die Entscheidungen im Bildungsverlauf. Dabei ist davon auszugehen, dass der unmittelbare, familiäre Einfluss im Laufe der Bildungskarriere abnimmt.

Geht es darum, Bildungsungleichheit erzeugende Mechanismen auf der Mikroebene $\mathrm{zu}$ untersuchen, haben sich die Theorie der primären und sekundären Herkunftseffekte (Boudon 1974) und deren Weiterentwicklungen (für eine Übersicht siehe Kristen 1999; Maaz et al. 2006) zur Erklärung von Bildungsungleichheiten nach sozialer Herkunft und Migrationshintergrund mehrfach empirisch bewährt (für eine 
Übersicht zu Deutschland vergleiche Dollmann 2016). Während unter primären Effekten für gewöhnlich der direkte Einfluss der sozialen Herkunft auf Schulleistungen, vermittelt über genetische, psychologische und/oder kulturelle Faktoren (Breen und Goldthorpe 1997), verstanden wird, bezeichnen sekundäre Effekte die subjektiven Kosten-Nutzen-Evaluationen, welche einer Bildungsentscheidung vorausgehen. Dabei spielt das Statuserhaltsmotiv eine zentrale Rolle, wonach Personen höhere Anstrengungen zu Vermeidung allfälliger Statusverluste für ihre Kinder aufbringen sollten, als um Statusgewinne zu erzielen. Das wiederum bedeutet, dass der Nutzen höherer Bildungsabschlüsse für den Statuserhalt (die sogenannte Instrumentalität) statushöherer Personen ceteris paribus grösser ist (Beck 2015; Glauser 2015; Stocké 2007). Darüberhinausgehender Nutzen von Bildungszertifikaten kann beispielsweise in höheren Einkommen oder einem geringeren Arbeitslosigkeitsrisiko liegen (Beck 2015; Glauser 2015; Jäpel 2017). Kosten können als direkte Ausbildungskosten oder entgangenes Einkommen angenommen werden. Zudem wird die Kosten-Nutzen-Evaluation durch die Einschätzung der Erfolgswahrscheinlichkeiten, einen gewählten Bildungsweg auch erfolgreich abzuschließen, beeinflusst (Beck 2015; Glauser 2015; Gresch 2012; Becker 2010; Stocké 2007).

Die primären sowie sekundären Herkunftseffekte werden zur Erklärung von migrationsbedingten Bildungsungleichheiten um eine migrationsbezogene ${ }^{5}$ Dimension erweitert (Beck et al. 2010; Dollmann 2010; Kristen und Dollmann 2010; Van De Werfhorst und Van Tubergen 2007). Primäre migrationsbedingte Herkunftseffekte beziehen sich auf Nachteile, welche meist durch eine von der Schulsprache unterschiedliche Herkunftssprache entstehen (zu einer ausführlichen Betrachtung der Rolle von Sprache auf den Bildungserfolg von Schülerinnen und Schülern mit Migrationshintergrund siehe Kempert et al. 2016), aber auch Unterschiede in der Erziehungskultur. Sekundäre migrationsbedingte Herkunftseffekte bezeichnen Unterschiede im Entscheidungsverhalten, die durch migrationsspezifische Kosten-Nutzen-Evaluationen von Bildungsalternativen entstehen.

Wie bereits gezeigt werden konnte, legt der aktuelle Forschungsstand nahe, dass sekundäre migrationsbedingte Effekte häufiger positiv sind, was bei vergleichbaren Schulleistungen und vergleichbarer sozialer Herkunft von Schülerinnen und Schüler mit Migrationshintergrund dazu führt, dass diese an Verzweigungspunkten im Bildungssystem tendenziell häufiger anspruchsvollere Bildungswege wählen. Auch für die Schweiz gibt es Befunde, dass Schülerinnen und Schüler mit Migrationshintergrund sowie deren Eltern höhere Bildungsaspirationen berichten. Zur Erklä-

5 Auch wenn bislang der Begriff „ethnische Herkunftseffekte“ häufig zur Anwendung kommt, so ist dieser unserer Ansicht nach problematisch, da er eine gewisse Homogenität unterschiedlichster Migrationsbiographien in Abhängigkeit der Herkunftsländer impliziert. Aus diesem Grund soll hier der weiter gefasste Begriff „migrationsbedingte Herkunftseffekte“ verwendet werden. Dieser Begriff vereinfacht die Heterogenität unterschiedlicher Migrationsmotive zwar immer noch stark, schreibt ihnen aber weniger ein gruppen- (bzw. „ethnien-“) spezifisches Element zu. 
rung werden unterschiedliche Mechanismen diskutiert (vergleiche zu den folgenden Punkten, wo nicht anders angegeben Becker 2010 sowie Becker und Gresch 2016):

1) Relative soziale Position (Van De Werfhorst und Van Tubergen 2007): Insbesondere Personen aus Ländern, welche ökonomisch geringer entwickelt sind und bei welchen die Bildungsexpansion noch nicht in dem gleichen Masse fortgeschritten ist, wie in west- und nordeuropäischen Ländern, sind im Vergleich zu formell gleich gebildeten Personen im Aufnahmeland überdurchschnittlich fähig. Dies bedeutet, dass ihr sozialer Status im Herkunftsland relativ gesehen höher war. Also geben sie höhere Bildungsaspirationen an ihre Kinder weiter, um den durch Migration erlittenen Statusverlust auszugleichen.

2) Immigrant Optimism (Kao und Tienda 1995): Personen mit Migrationshintergrund stellen eine nach Ambitionen und Aspirationen positiv selektionierte Gruppe dar, da ein zentrales Motiv ihrer Migration häufig ein Streben nach Verbesserung ihrer Lebenschancen und denen ihrer Kinder war.

3) (Antizipierte) Diskriminierung (Heath und Brinbaum 2007): Sollten Personen mit Migrationshintergrund befürchten, dass ihre Kinder später im Bewerbungsprozess für ein berufliche Ausbildung ${ }^{6}$ oder im Arbeitsleben diskriminiert werden, so kann einerseits versucht werden, dies durch höhere Bildungsaspirationen und damit verbundene Mehrinvestitionen in Bildungszertifikate zu kompensieren. Andererseits kann die Konsequenz ein längeres Verweilen im Bildungssystem sein, um den Eintritt in den Arbeitsmarkt und die damit verbundene (erwartete) Diskriminierung weiter hinauszuzögern.

4) Informationsmängel: Diese liegen in mangelnden Kenntnissen der institutionellen Rahmenbedingungen und der Schulsysteme durch Personen mit Migrationshintergrund begründet, wodurch die Wahrscheinlichkeiten für einen erfolgreichen Abschluss anspruchsvollerer Bildungslaufbahnen überschätzt werden. Eine derartige Überschätzung kann auch in Bezug auf die Schulleistungen der eigenen Kinder passieren (Gresch 2012).

Während sekundäre Herkunftseffekte überwiegend Einschätzungen und Entscheidungen auf der Mikroebene betreffen, sind die Rahmenbedingungen, welche das Entscheidungsverhalten beeinflussen, eher auf der Mesoebene und der Makroebene angesiedelt. Da der Fokus dieses Artikels auf der Mikroebene liegt, wird im Folgenden nur kurz auf diese beiden Ebenen eingegangen.

Auf der Mesoebene sind Effekte der Zusammensetzung von Schulklassen ein vermehrt untersuchtes Thema, vor allem in Bezug auf schulische Lernprozesse (Ditton 2007; Schallock 2016). Hier werden neben Kontexteffekten der Klassenzusammenset-

6 In Ländern mit hoher beruflicher Spezifität und einem stark ausgeprägten beruflichen Ausbildungssystem wie in der Schweiz sind Bewerbungsverfahren bereits beim Eintritt in die Berufslehre typisch. 
zung $^{7}$ die Erwartungen und daraus resultierenden Bildungsempfehlungen von Lehrpersonen untersucht, welche häufig als ,tertiäre Herkunftseffekte“ bezeichnet werden. Da Lehrpersonen nicht nur bei der Unterrichtsgestaltung und der Kompetenzvermittlung, sondern auch bei der Notenvergabe und Übertrittsempfehlung eine zentrale Rolle zukommt, werden sie in der Forschung als potentielle Quelle für Diskriminierung betrachtet ${ }^{8}$. Dabei bestehen unterschiedliche Konzepte von Diskriminierung (Diehl und Fick 2016). Statistische Diskriminierung (Arrow 1985) meint ungleiche Bewertung der Kompetenzen von Angehörigen einer Minderheit auf Grund von Informationsmängeln und dem daraus entstehenden Zurückgreifen auf eine Heuristik über deren Leistungsfähigkeit. Durch die tägliche Begegnung mit ihren Schülerinnen und Schülern können Lehrpersonen jedoch auf die tatsächlich gezeigte Leistung zurückgreifen und müssen nicht nach Heuristiken bewerten, weshalb diese Art der Diskriminierung eher unwahrscheinlich ist, wie auch Kristen (2006) für Deutschland und Becker et al. (2013) für die Schweiz zeigen können. Zumindest theoretisch wahrscheinlicher sind eine präferenzbasierte Diskriminierung (Becker 1973), ein bewusstes schlechteres Bewerten auf Grund des Migrationshintergrund der Schülerinnen und Schüler oder eine indirekte institutionelle Diskriminierung (Gomolla und Radtke 2000), welche auf Normalitätserwartungen der im Bildungssystem handelnden Akteure basiert und beispielsweise geringere Kenntnisse der Schulsprache als Bedarf für sonderpädagogische Maßnahmen interpretiert (Gomolla und Radtke 2000; Kronig 2003; Kronig et al. 2000).

Auf der Makroebene spielen zum Beispiel rechtliche Faktoren (zum Einfluss des Ausländerrechts siehe Söhn 2011), die Ausgestaltung des Bildungssystems (vergleiche Ditton in diesem Band), wie die Form des Trackings (Schallock 2016), sowie das Angebot an vorschulischer Bildung und Sprachförderung (Bauer und Riphahn 2010; Fritschi und Jann 2009) eine Rolle. Im Bereich des Übergangs zum Arbeitsmarkt sind wiederum ausländerrechtliche Faktoren, aber auch alternative Opportunitätsstrukturen im Sinne von ethnic business (Integrationsförderung Stadt Zürich 2008) von Bedeutung, welche allenfalls keine standardisierten Bildungszertifikate voraussetzen und alternative Erwerbsmöglichkeiten darstellen können.

Nach der obligatorischen Schulzeit gibt es unterschiedliche Faktoren, welche bei Schülerinnen und Schülern mit Migrationshintergrund dazu führen können, dass keine nachobligatorische Ausbildung begonnen wird. Diese können nach Hunkler $(2014 ; 2016)$ in soziale, migrationsspezifische und ethnische Faktoren unterteilt werden. Unter sozialen Faktoren werden hier Einflüsse des sozialen Hintergrunds verstanden, welche sich grundsätzlich an den oben skizierten primären und sekundä-

7 Der Anteil an Schülerinnen und Schülern mit Migrationshintergrund hat nach dem momentanen Forschungsstand unter Kontrolle des sozioökonomischen Klassenkontextes keinen eigenständigen Einfluss auf Übertritte (Trautwein und Baeriswyl 2007).

8 Dass dies aus theoretischen und statistischen Gründen nicht immer unproblematisch ist, zeigt Hunkler (2014). 
ren Effekten orientieren und entsprechend bei autochthonen Personen identisch sind. Unter migrationsspezifischen Faktoren werden Fähigkeiten und Präferenzen verstanden, die durch die Migrationssituation beeinflusst sind (zum Beispiel das Fehlen bestimmter berufsrelevanter Kompetenzen, Uninformiertheit bezüglich des Ausbildungs- und Arbeitsmarktsystems, unvorteilhafte Berufswünsche sowie höhere Aspirationen, welche zu einem Übertritt auf eine anspruchsvollere Schulform führen $^{9}$ ), sowie ethnischen Faktoren, im Sinne von Diskriminierung durch Arbeitgeberinnen und Arbeitgeber. Im Gegensatz zur schulischen Situation ist statistische Diskriminierung von Bewerberinnen und Bewerbern mit Migrationshintergrund eher plausibel (vergleiche im Folgenden, wo nicht anders angegeben, Beck et al. 2010), da im Bewerbungsprozess auf Grund des Mangels an verlässlichen Informationen über die Eigenschaften von Personen meist auf Signale (Spence 1973) zurückgegriffen wird. Zusätzlich zu den Schulabschlüssen können Ausbildungsbetriebe auf askriptive Merkmale der Lehrstellenbewerbenden, wie etwa einen Migrationshintergrund, zurückgreifen. In der Logik der statistischen Diskriminierung würde bei den Auswahlentscheidungen auf Grund einer bestimmen Gruppenzugehörigkeit auf das Verhalten einzelner Personen geschlossen. Ein bei Personen mit Migrationshintergrund relativ häufiger vorhandener niedrigerer Schulabschluss (Meyer et al. 2003; BFS 2017b) könnte zudem als geringere Leistungsfähigkeit oder Zuverlässigkeit interpretiert werden. Es würde möglicherweise ein organisatorischer Mehraufwand bei Sprachproblemen erwartet, sowie Unsicherheit über einen längerfristigen Verbleib ausländischer Auszubildenden und die damit verbundene Gefahr geringerer Verwertungsgrade bis hin zu Fehlinvestitionen (Seibert und Solga 2005). Es kann also nicht ausgeschlossen werden, dass Betriebe den Signalwert von anspruchsärmeren Schulabschlüssen zusätzlich ethnisch modifizieren und somit Personen mit Migrationshintergrund im Allgemeinen und bestimmte Nationalitäten im Besonderen bei den Chancen für eine duale Berufsausbildung im Nachteil sind (Hupka et al. 2006).

\section{Empirische Ergebnisse}

Die empirischen Analysen erfolgen in zwei Schritten. Zunächst untersuchen wir die Platzierung von Schülerinnen und Schülern mit Migrationshintergrund in die Sekundarstufe I. Danach gehen wir der Frage nach, wie groß das Risiko für Jugendliche mit Migrationshintergrund im Vergleich zu den Einheimischen ist, am Ende der obligatorischen Schule nicht direkt in eine zertifizierende Sekundar-II-Ausbildung einzutreten $^{10}$.

9 Dabei kann es vorkommen, dass unrealistisch überhöhte Bildungsaspirationen eher dazu führen, dass die gewählte Schulform nicht erfolgreich abgeschlossen wird.

10 Ähnliche und weitergehende Analysen mit denselben Daten finden sich bei Beck (2015), Glauser (2015; 2018) sowie bei Jäpel (2017). 


\section{Vorgehen und Datenbasis}

Bei der Frage, ob und welche Migrationsgruppen beim Übertritt von der Primar- in die Sekundarstufe I ein besonders hohes Risiko haben, nach der sechsten Primarklasse auf Schulformen mit Grundanforderungen überwiesen zu werden, soll insbesondere überprüft werden, inwieweit die Überweisungen nach leistungsgerechten Kriterien erfolgen und ob eine Benachteiligung von Schulkindern mit Migrationshintergrund festzustellen ist. Hierfür ziehen wir Daten der DEBIMISS-Studie (Determinanten des Bildungserfolgs von Migranten im Schweizer Schulsystem) heran (Beck 2015). Dabei wurden zwischen 2009 und 2011 Schülerinnen und Schüler sowie deren primäre Bezugspersonen aus Primarschulen der Deutschschweizer Städte Bern und Zürich ein Jahr vor und unmittelbar nach dem Übertritt von der Primar- auf die Sekundarstufe untersucht. Insgesamt stehen für die Analysen 520 Datensätze zur Verfügung ${ }^{11}$.

Für die Analyse des Risikos, am Ende der obligatorischen Schulzeit nicht direkt in eine zertifizierende Sekundar-II-Ausbildung einzutreten, werden die Daten der Schweizer Panelstudie DAB (Determinanten der Ausbildungsbildungswahl und der Berufsbildungschancen) verwendet (Jäpel 2017; Glauser 2015). DAB ist eine Längsschnittuntersuchung im Panel-Design, welche die Übertritte von mehr als 3000 Sekundarschülerinnen und Sekundarschülern der Schulabgangskohorte 2013 für die deutschsprachige Schweiz untersucht.

In den nachfolgenden Analysen werden verschiedene Merkmale der Schulkinder einbezogen, die im Zusammenhang mit den genannten Übertrittsrisiken stehen. Neben dem Geschlecht sind dies die schulische Leistung (operationalisiert über den Mittelwert der Jahresdurchschnittsnoten in Deutsch und Mathematik in der sechsten respektive der achten Klasse) sowie die Herkunft der Jugendlichen. Zur Abbildung des Migrationshintergrundes werden der Geburtsort des ausfüllenden Elternteils (DEBIMISS) respektive des Vaters des befragten Jugendlichen (DAB) sowie die Sprache, von der die befragten Personen angeben, dass sie diese zu Hause am häufigsten sprechen, verwendet. Zum einen wird also eine allfällige Fremdsprachigkeit in der Herkunftsfamilie berücksichtigt und zum anderen werden die Personen je nach nationaler Herkunft in Migrationswellen eingeteilt. Wie bereits in anderen Studien beschrieben (Beck et al. 2010), wird zwischen Angehörigen der historisch zweiten Migrationswelle (ehemaliges Jugoslawien, Albanien, Türkei, Portugal sowie Sri Lanka) und Angehörigen anderer Länder unterschieden. Die Geburt des Elternteils der befragten Personen in einem deutschsprachigen Land (Schweiz inclusive Lichtenstein, Deutschland und Österreich) und (schweizer-)deutsche Sprache sind die jeweiligen Referenzkategorien.

11 Fehlende Werte wurden mit der Methode der Chained Equations (White et al. 2011) imputiert $(\mathrm{M}=50)$. 
Die soziale Herkunft wird folgendermaßen operationalisiert: Für die Klassenlage wird das EGP-Klassenschema von Erikson et al. (1979) verwendet. Für die Analysen wurden die Kategorien I-VII gebildet und in einer Variable mit den Kategorien I, II, III-IV und V-VII zusammengefasst. Aus den Angaben zum Bildungshintergrund der Eltern wird der höchste im Familienhaushalt vorhandene Bildungsabschluss herangezogen und über die Zuordnung zur ISCED-97-Klassifikation operationalisiert. Unterschieden werden Abschlüsse bis maximal ISCED 2 (maximal obligatorische Ausbildung), Abschlüsse der Kategorien ISCED 3-4 (Abschluss der Sekundarstufe II) und die dritte Gruppe mit mindestens einem tertiären Abschluss in der Familie bzw. ISCED 5-6 (tertiäre Bildungsabschlüsse). Die Referenzkategorien bilden jeweils die höchsten Kategorien (Obere Dienstklasse (I) bzw. tertiäre Abschlüsse). Für den ersten Übertritt werden außerdem sekundäre Herkunftseffekte berücksichtigt, dabei handelt es sich um die elterliche Einschätzung der oben genannten Kosten-Nutzen-Evaluationen. Diese wird ermittelt durch die Differenz der elterlichen Einschätzungen der Instrumentalität, der (direkten und indirekten) Kosten, des erwarteten Nutzens (zukünftiges Einkommen und Arbeitslosigkeitsrisiko) sowie der Erfolgswahrscheinlichkeit des jeweiligen Abschlusses. Ein positiver Wert der Variable bedeutet, dass die Einschätzung zu Gunsten des anspruchsvolleren Niveaus auf Sekundarstufe I ausfällt (zu den genauen Operationalisierungen vergleiche Beck 2015).

Für die statistischen Analysen werden binäre und multinomiale logistische Regressionen geschätzt (Long 1997; Long und Freese 2014). Um die Ergebnisse der verschiedenen Modelle vergleichen zu können, werden entsprechend der Empfehlung von Best und Wolf (2012) Average Marginal Effects (AME) ausgewiesen. Diese drücken die durchschnittlichen Effekte der unabhängigen Variablen auf die Eintrittswahrscheinlichkeit eines Ereignisses in einer einzigen Kennziffer aus und haben den Vorteil, dass sie von unbeobachteter Heterogenität nicht verzerrt werden.

\section{Übertritte von der Primarschule in die Sekundarstufe I}

In Tabelle 1 sind die Übertritte von der Primar- auf die Sekundarstufe I für die in DEBIMISS untersuchten Gemeinden Bern und Zürich dargestellt. Abhängige Variable ist das Risiko, in die Sekundarschule mit Grundanforderungen einzutreten. Es zeigt sich, dass unter Kontrolle der sozialen Herkunft sowie der Schulnoten kein Einfluss des Migrationshintergrundes und der Sprache auf den Übertritt besteht. Grundsätzlich haben nur die Schulnoten sowie die Schulgemeinde einen nennenswerten Einfluss.

Die Wahrscheinlichkeit, auf die anspruchsvolleren Niveaus der Sekundarstufe I zu wechseln ist in Zürich um 20 Prozent höher als in Bern. Es zeigt sich, dass die Übertritte überwiegend durch die Schulleistung strukturiert werden, das Übertrittsrisiko auf die Realschule ist bei einer Abweichung der durchschnittlichen Schulleistung um eine Standardabweichung nach unten um etwa 20 Prozent geringer. Die migrations- 
Tabelle 1 Einflussfaktoren auf das Risiko des Übertrittes nach der Primarstufe in eine Schule mit Grundanforderungen (logistische Regression)

\begin{tabular}{|c|c|c|}
\hline & Mod.1: Übertritt & $\begin{array}{l}\text { Mod.2: Übertritt } \\
\text { Sekundäre Effekte }\end{array}$ \\
\hline \multirow[t]{2}{*}{ Schulleistung } & $-0.21^{* * *}$ & $-0.19 * * *$ \\
\hline & $(0.007)$ & $(0.011)$ \\
\hline \multirow[t]{2}{*}{ Zürich } & $-0.21^{* * *}$ & $-0.18^{* * *}$ \\
\hline & $(0.025)$ & $(0.026)$ \\
\hline \multirow[t]{2}{*}{ Mädchen } & -0.00 & 0.03 \\
\hline & $(0.018)$ & $(0.017)$ \\
\hline \multirow[t]{2}{*}{ Fremdsprachig } & 0.02 & 0.03 \\
\hline & $(0.029)$ & $(0.027)$ \\
\hline \multicolumn{3}{|l|}{$\begin{array}{l}\text { Migrationshintergrund } \\
\text { (Ref.: } \mathrm{CH} / \mathrm{D} / \mathrm{O})\end{array}$} \\
\hline \multirow[t]{2}{*}{ 2. Migrationswelle } & 0.00 & 0.01 \\
\hline & $(0.029)$ & $(0.027)$ \\
\hline \multirow[t]{2}{*}{ Andere Länder } & -0.02 & -0.01 \\
\hline & $(0.031)$ & $(0.032)$ \\
\hline \multicolumn{3}{|l|}{$\begin{array}{l}\text { Klassenlage Eltern } \\
\text { (Ref.: EGP I) }\end{array}$} \\
\hline \multirow[t]{2}{*}{ EGP ॥ } & -0.05 & -0.06 \\
\hline & $(0.044)$ & $(0.042)$ \\
\hline \multirow[t]{2}{*}{$\mathrm{EGP} \| \mathrm{ll} \mathrm{a} / \mathrm{b}, \mathrm{IVa} / \mathrm{b} / \mathrm{c}$} & -0.01 & -0.02 \\
\hline & $(0.035)$ & $(0.035)$ \\
\hline \multirow[t]{2}{*}{ EGP V, VI, VIla/b } & -0.02 & -0.02 \\
\hline & $(0.031)$ & $(0.029)$ \\
\hline \multicolumn{3}{|l|}{$\begin{array}{l}\text { Elterlicher Bildungsstand } \\
\text { (Ref.: ISCED 5-6) }\end{array}$} \\
\hline \multirow[t]{2}{*}{ max. ISCED 2} & 0.03 & 0.02 \\
\hline & $(0.031)$ & $(0.033)$ \\
\hline \multirow[t]{2}{*}{ ISCED 3-4 } & $0.06^{* *}$ & $0.05^{*}$ \\
\hline & $(0.020)$ & $(0.023)$ \\
\hline \multicolumn{3}{|l|}{ Sekundäre Effekte } \\
\hline \multirow[t]{2}{*}{ Instrumentalität } & & -0.01 \\
\hline & & $(0.009)$ \\
\hline \multirow[t]{2}{*}{ Kosten } & & -0.01 \\
\hline & & $(0.009)$ \\
\hline \multirow[t]{2}{*}{ Erfolgswahrscheinlichkeit } & & $-0.02 *$ \\
\hline & & $(0.008)$ \\
\hline \multirow[t]{2}{*}{ Nutzen } & & 0.01 \\
\hline & & $(0.011)$ \\
\hline Pseudo $R^{2}$ & 0.71 & 0.72 \\
\hline Beobachtungen & 520 & 520 \\
\hline
\end{tabular}

Daten: DEBIMISS, eigene Berechnungen und Darstellung.

Hinweise: Average Marginal Effects; robuste Standardfehler in Klammern; Schulklassen als Cluster; Fehlende Werte imputiert $(M=50) ;{ }^{+} p<0.10,{ }^{*} p<0.05,{ }^{* *} p<0.01,{ }^{* * *} p<0.001$ 
spezifischen Variablen zeigen keine nennenswerten Effekte. Für die hier untersuchte Stichprobe ist eine Ungleichbehandlung von Schülerinnen und Schülern mit Migrationshintergrund an der ersten Schwelle empirisch nicht nachweisbar. Eine Diskriminierung durch Lehrpersonen scheint somit, zumindest beim ersten Übertritt, nicht gegeben. Gleichzeitig liefern sekundäre Herkunftseffekte unter der Kontrolle der Schulleistungen hier nur noch einen marginalen Beitrag. Auch kann gezeigt werden, dass das Risiko auf Schulformen mit Grundanforderungen überzugehen stark mit dem institutionellen Kontext variiert.

\section{Übertritte am Ende der Sekundarstufe I}

Im zweiten Schritt werden die Übertrittschancen am Ende der Sekundarstufe I untersucht $^{12}$. Für Personen mit Migrationshintergrund wird im Vergleich zu den Einheimischen ein signifikant höheres Risiko angenommen, nach der Pflichtschulzeit nicht direkt in eine zertifizierende Ausbildung einzutreten. Infolgedessen laufen sie eher Gefahr, ausbildungslos zu bleiben, aufgrund von Bildungsarmut Erfahrungen mit häufiger oder langanhaltender Arbeitslosigkeit zu machen und allenfalls geringere Einkommen zu erzielen. Anhand dieses Risikos für eine Zertifikatsarmut soll überprüft werden, ob Personen mit Migrationshintergrund hier besonders benachteiligt sind. In der Stichprobe befinden sich insgesamt 2036 Personen mit gültigen Werten auf allen interessierenden Variablen.

Die Analysen erfolgen in zwei Schritten. Zunächst wird ein Modell geschätzt, in dem keine Unterscheidung zwischen beruflichen und allgemeinbildenden Ausbildungsformen vorgenommen wird (Tabelle 2). Die abhängige Variable ist das Risiko für Zertifikatsarmut in der Sekundarstufe II, also über keine Zusage für einen zertifizierenden Ausbildungsweg am Ende der neunten Klasse - und damit wenige Wochen vor Ende der obligatorischen Schulzeit - zu verfügen. Die Referenzkategorie sind diejenigen Personen, die zu diesem Zeitpunkt eine Zusage für eine Berufslehre mit/ohne Berufsmaturitätsschule oder den Besuch einer Mittelschule haben. Den Schätzergebnissen für das Modell 1 in Tabelle 2 zufolge haben - unter Kontrolle der schulischen Leistungen, des in der Sekundarstufe II besuchten Schultyps sowie des Geschlechts - Jugendliche mit Migrationshintergrund ein signifikant höheres Risiko als Jugendliche, deren Eltern aus der Schweiz oder den deutschsprachigen Nachbarländern stammen, am Ende der neunten Klasse noch keine Zusage für eine zertifizierende nachobligatorische Ausbildung zu haben und damit dem Risiko für Zertifikatsarmut ausgesetzt zu sein. Dies gilt insbesondere für die Gruppe derjenigen Personen

12 Zwei Personengruppen wurden aus den Analysen ausgeschlossen: Zum einen sind das diejenigen Jugendlichen, die zum Befragungszeitpunkt ein Gymnasium besuchen (und damit den interessierenden Übertritt bereits erfolgreich absolviert haben) und solche, die eine Zusage für eine nicht zertifizierende Zwischenlösung haben (und bei denen sich der interessierende Übertritt vorerst verschiebt). 
Tabelle 2 Übertritte von der Sekundarstufe I in die Sekundarstufe II (logistische Regression)

\begin{tabular}{|c|c|c|}
\hline & $\begin{array}{l}\text { Mod.1: Keine zertifizierte } \\
\text { Sek-II Ausbildung }\end{array}$ & $\begin{array}{l}\text { Mod. 2: Keine zertifizierte } \\
\text { Sek-II Ausbildung }\end{array}$ \\
\hline \multirow[t]{2}{*}{ Schulleistung } & $-0.04^{* *}$ & $-0.04^{*}$ \\
\hline & $(0.014)$ & $(0.014)$ \\
\hline \multicolumn{3}{|l|}{$\begin{array}{l}\text { Schultyp Sek. I } \\
\text { (Ref.: Grundanforderungen) }\end{array}$} \\
\hline \multirow[t]{2}{*}{ erweiterte Anforderungen } & $-0.04^{*}$ & $-0.04^{*}$ \\
\hline & $(0.017)$ & $(0.017)$ \\
\hline \multirow[t]{2}{*}{ Ohne Selektion } & 0.00 & 0.01 \\
\hline & $(0.037)$ & $(0.036)$ \\
\hline \multirow[t]{2}{*}{ Frauen } & $0.03^{*}$ & $0.04^{* *}$ \\
\hline & $(0.013)$ & $(0.013)$ \\
\hline \multirow[t]{2}{*}{ Fremdsprachig } & $-0.06^{*}$ & $-0.06^{*}$ \\
\hline & $(0.022)$ & $(0.021)$ \\
\hline \multicolumn{3}{|l|}{$\begin{array}{l}\text { Migrationshintergrund } \\
\text { (Ref.: } \mathrm{CH} / \mathrm{D} / \mathrm{O})\end{array}$} \\
\hline \multirow[t]{2}{*}{ 2. Migrationswelle } & $0.05^{*}$ & $0.05^{*}$ \\
\hline & $(0.021)$ & $(0.023)$ \\
\hline \multirow[t]{2}{*}{ Übrige Länder } & $0.10^{* * *}$ & $0.10^{* * *}$ \\
\hline & $(0.024)$ & $(0.023)$ \\
\hline \multicolumn{3}{|l|}{$\begin{array}{l}\text { Klassenlage Eltern } \\
\text { (Ref.: EGP I) }\end{array}$} \\
\hline \multirow[t]{2}{*}{ EGP II } & & 0.01 \\
\hline & & $(0.024)$ \\
\hline \multirow[t]{2}{*}{ EGP IIIa/b, IVa/b/c } & & 0.01 \\
\hline & & $(0.024)$ \\
\hline \multirow[t]{2}{*}{ EGP V, VI, VIla/b } & & -0.00 \\
\hline & & $(0.022)$ \\
\hline \multicolumn{3}{|l|}{$\begin{array}{l}\text { Elterlicher Bildungsstand } \\
\text { (Ref.: ISCED 5-6) }\end{array}$} \\
\hline \multirow[t]{2}{*}{ ISCED 3-4 } & & -0.01 \\
\hline & & $(0.019)$ \\
\hline \multirow[t]{2}{*}{ ISCED 1-2 } & & -0.02 \\
\hline & & $(0.023)$ \\
\hline Pseudo $R^{2}$ & 0.04 & 0.05 \\
\hline Beobachtungen & 2036 & 2036 \\
\hline
\end{tabular}

Daten: DAB-Panel, eigene Berechnungen und Darstellung.

Hinweise: Average Marginal Effects; robuste Standardfehler in Klammern; Schulklassen als Cluster; kontrolliert für fehlende Werte in EGP und ISCED; ${ }^{+} p<0.10,{ }^{*} p<0.05,{ }^{* *} p<0.01,{ }^{* * *} p<0.001$ 
mit Migrationshintergrund, die nicht der zweiten Migrationswelle zuzuordnen sind. Interessant sind hingegen die Effekte einer allfälligen Fremdsprachigkeit in der Familie, hier kehrt sich der Effekt um: Fremdsprachige Jugendliche zeigen am Übergang in die Sekundarstufe II ein signifikant geringeres Risiko für Zertifikatsarmut. Dieser Befund geht jedoch mit den eingangs skizzierten Annahmen der Theorie zur Relativen soziale Position (Van de Werfhorst und van Tubergen 2007) sowie der Immigrant Optimism Hypothesis (Kao und Tienda 1995) einher, wonach sich überdurchschnittliche Fähigkeiten bei gleichzeitig (noch) nicht formalisierten Abschlüssen der Eltern zusammen mit dem Streben nach einer Verbesserung der eigenen Lebensbedingungen bestimmter Migrationsgruppen in höheren Bildungsaspirationen und -anstrengungen ihrer Kinder niederschlagen.

Um zu überprüfen, ob sich die negativen Effekte des Migrationshintergrundes auf die soziale Herkunft der Jugendlichen zurückführen lassen und das erhöhte Risiko für Zertifikatsarmut durch Migration als Spezialfall der sozialen Herkunft (Kalter 2008) erklären lässt, werden in Modell 2 für die soziale Herkunft die Klassenlage des Elternhauses (EGP-Klasse) sowie der höchste Bildungsabschluss im Elternhaus (ISCED) berücksichtigt. Die Effekte sind jedoch allesamt insignifikant, so dass diese These für das frühe Risiko für absolute Zertifikatsarmut nicht bestätigt werden kann. Der Migrationshintergrund an sich bringt, wie der Status einer Person mit Migrationshintergrund der zweiten Migrationswelle, offensichtlich Nachteile für die betreffenden Jugendlichen mit sich, nach Schulabschluss nicht direkt in eine zertifizierende Ausbildung einzutreten.

Um Unterschiede in der Art des zugesagten Ausbildungsweges zu untersuchen, wurden in einer weiteren Analyse mittels multinomialer logistischer Regression die Wahrscheinlichkeit einer Zusage für eine klassische Berufslehre, für eine Ausbildung, die zu einer Hochschulberechtigung führt (Gymnasium, Mittelschule, Lehre mit Besuch der Berufsmaturitätsschule) dem Risiko der Zertifikatsarmut (keine Zusage für eine zertifizierende Ausbildung) gegenübergestellt (Tabelle 3).

Hier zeigt sich, dass Jugendliche mit Migrationshintergrund nicht nur eine geringere Chance auf einen direkten Eintritt in nachobligatorisch zertifizierende Ausbildungen haben, darüber hinaus haben sie geringere Chancen, eine Zusage für eine Berufslehre $\mathrm{zu}$ erhalten. Diese Hinweise untermauern die signaltheoretische Perspektive, der zufolge ausbildende Betriebe den Migrationshintergrund und Sprachfertigkeiten als askriptive screening devices für effiziente Trainierbarkeit und Trainingskosten verwenden würden. Da für den Migrationshintergrund keine erhöhte Wahrscheinlichkeit in die Allgemeinbildung überzugehen festgestellt werden kann, spricht dies eher gegen zu hohe Bildungsaspirationen, zumindest von Personen mit Migrationshintergrund der zweiten Migrationswelle. Die Effekte der sozialen Herkunft werden nun bei der Unterscheidung nach berufs- und allgemeinbildenden Bildungswegen deutlich, wonach Kinder aus den höheren Klassen und mit mindestens einem tertiär gebildeten Elternteil höhere Chancen haben, eine Zusage für eine Ausbildung mit dem Ziel einer Hochschulzugangsberechtigung zu erhalten. 
Tabelle 3 Übertritte von der Sekundarstufe I in die Sekundarstufe II (multinomiale logistische Regression)

\begin{tabular}{|c|c|c|c|}
\hline & Keine Ausbildung & $\begin{array}{l}\text { Zusage Berufs- } \\
\text { ausbildung }\end{array}$ & $\begin{array}{l}\text { Zusage Matur } \\
\text { (Gym./Beruf) }\end{array}$ \\
\hline \multirow[t]{2}{*}{ Schulleistung } & $-0.04^{* *}$ & $-0.10^{* * *}$ & $0.13^{* * *}$ \\
\hline & $(0.014)$ & $(0.022)$ & $(0.021)$ \\
\hline \multicolumn{4}{|l|}{$\begin{array}{l}\text { Schultyp Sek. I } \\
\text { (Ref.: Grundanforderungen) }\end{array}$} \\
\hline \multirow[t]{2}{*}{ erweiterte Anforderungen } & $-0.04^{*}$ & -0.04 & $0.08^{* * *}$ \\
\hline & $(0.017)$ & $(0.030)$ & $(0.025)$ \\
\hline \multirow[t]{2}{*}{ Ohne Selektion } & 0.00 & $-0.09 *$ & $0.09 *$ \\
\hline & $(0.036)$ & $(0.047)$ & $(0.046)$ \\
\hline \multirow[t]{2}{*}{ Frauen } & $0.04 * *$ & -0.01 & -0.02 \\
\hline & $(0.013)$ & $(0.023)$ & $(0.021)$ \\
\hline \multirow[t]{2}{*}{ Fremdsprachig } & $-0.06^{* *}$ & -0.01 & $0.06^{+}$ \\
\hline & $(0.021)$ & $(0.040)$ & $(0.034)$ \\
\hline \multicolumn{4}{|l|}{$\begin{array}{l}\text { Migrationshintergrund } \\
\text { (Ref.: } \mathrm{CH} / \mathrm{D} / \mathrm{O} \text { ) }\end{array}$} \\
\hline \multirow[t]{2}{*}{ 2. Migrationswelle } & $0.05^{*}$ & -0.05 & -0.00 \\
\hline & $(0.023)$ & $(0.036)$ & $(0.031)$ \\
\hline \multirow[t]{2}{*}{ Übrige Länder } & $0.09 * * *$ & $-0.09 *$ & 0.01 \\
\hline & $(0.023)$ & $(0.036)$ & $(0.036)$ \\
\hline \multicolumn{4}{|l|}{$\begin{array}{l}\text { Klassenlage Eltern } \\
\text { (Ref.: EGP I) }\end{array}$} \\
\hline \multirow[t]{2}{*}{ EGP ॥ } & 0.01 & 0.06 & $-0.07^{+}$ \\
\hline & $(0.024)$ & $(0.038)$ & $(0.036)$ \\
\hline \multirow[t]{2}{*}{$\mathrm{EGP} \| \mathrm{lla} / \mathrm{b}, \mathrm{IVa} / \mathrm{b} / \mathrm{c}$} & 0.01 & $0.10^{*}$ & $-0.10^{* *}$ \\
\hline & $(0.024)$ & $(0.039)$ & $(0.037)$ \\
\hline \multirow[t]{2}{*}{ EGP V, VI, VIIa/b } & -0.00 & $0.10^{*}$ & $-0.09^{*}$ \\
\hline & $(0.022)$ & $(0.039)$ & $(0.037)$ \\
\hline \multicolumn{4}{|l|}{$\begin{array}{l}\text { Elterlicher Bildungsstand } \\
\text { (Ref.: ISCED 5-6) }\end{array}$} \\
\hline \multirow[t]{2}{*}{ ISCED 3-4 } & -0.02 & $0.10^{* * *}$ & $-0.10^{* *}$ \\
\hline & $(0.019)$ & $(0.025)$ & $(0.024)$ \\
\hline \multirow[t]{2}{*}{ ISCED 1-2 } & -0.02 & $0.13^{* * *}$ & $-0.11^{* * *}$ \\
\hline & $(0.023)$ & $(0.032)$ & $(0.031)$ \\
\hline Pseudo $R^{2}$ & & 0.05 & \\
\hline Wald- $x^{2}$ & & $156.44^{* * *}$ & \\
\hline Beobachtungen & & 2036 & \\
\hline
\end{tabular}

Daten: DAB-Panel, eigene Berechnungen und Darstellung.

Hinweise: Average Marginal Effects; robuste Standardfehler in Klammern; Schulklassen als Cluster; kontrolliert für fehlende Werte in EGP und ISCED; ${ }^{+} p<0.10,{ }^{*} p<0.05,{ }^{* *} p<0.01,{ }^{* * *} p<0.001$ 
Die Effekte der schulischen Leistungen und des besuchten Schultyps stehen zwar nicht im Fokus, jedoch sollen sie aufgrund ihrer Ausprägungen nicht unkommentiert bleiben. Gute schulische Leistungen verringern zum einen das Risiko für eine frühe Zertifikatsarmut signifikant und zum anderen erhöhen sie die Chance für eine $\mathrm{Zu}$ sage in anspruchsvolleren Ausbildungsformen. Dies geht mit den bisherigen Befunden zu den primären Effekten der sozialen Herkunft einher und bestätig außerdem die folgenreiche Wirkung der frühen und von sekundären Herkunftseffekten beeinflussten Selektion am Ende der Primarstufe zu Ungunsten von leistungsschwächeren Schulkindern. Bei einer Selektion in den Schultyp mit Grundanforderungen sind diese Schülerinnen und Schüler einem höheren Risiko ausgesetzt, nicht direkt in eine zertifizierende nachobligatorische Ausbildung eintreten zu können und bildungsarm im Sinne von Zertifikatsarmut zu werden.

\section{Fazit}

Basierend auf dem Forschungsstand und den hier gezeigten Analysen kann folgendes geschlossen werden: Unter der Einschränkung kategorisierender (und damit ein Stück weit künstlicher) Operationalisierung des Migrationshintergrunds und dem daraus resultierenden Problem der Verallgemeinerbarkeit kausaler Mechanismen, zeigt sich, dass Schülerinnen und Schüler mit Migrationshintergrund im Schweizer Schulsystem an beiden untersuchten Übertrittszeitpunkten ein erhöhtes Risiko haben, auf Pfade mit einem höheren Risiko für Bildungsarmut zu gelangen. Zunächst einmal legen die Befunde von Kronig et al. (2000) nahe, dass Schülerinnen und Schüler mit Migrationshintergrund häufiger an Sonderschulen überwiesen werden, als es auf Grund ihrer kognitiven Fähigkeiten zu erwarten wäre und somit Hinweise vorliegen, dass Sprachschwierigkeiten im Sinne der indirekten institutionellen Diskriminierung (Gomolla und Radtke 2000) als Lernschwierigkeiten umgedeutet werden.

Im Regelschulbetrieb scheinen am Übergangszeitpunkt von der Primar- auf die Sekundarschule Schülerinnen und Schüler mit Migrationshintergrund bei vergleichbaren Schulleistungen nicht explizit benachteiligt $\mathrm{zu}$ werden, primäre Herkunftseffekte sowie institutionelle Rahmenbedingungen strukturieren die hier untersuchten Übertritte zum großen Teil, wie die vorliegenden Analysen mit Übertrittsdaten aus Bern und Zürich zeigen und was auch durch andere Studien bestätigt werden kann. Es gibt jedoch auch Grund zur Annahme, dass die durchaus vorhandenen primären migrationsbedingten Herkunftseffekte (Moser et al. 2011) durch sekundäre migrationsbedingte Herkunftseffekte kompensiert werden. Dabei gilt es zu beachten, dass die Übertrittsverfahren in den meisten Schweizer Kantonen relativ standardisiert ablaufen und dementsprechend, zumindest an diesem Übergang, ein Migrationshintergrund durchaus nach der Interpretation von Kalter (2008) als Spezialfall der sozialen Herkunft gewertet werden kann. 
Beim Übertritt in nachobligatorische Ausbildungen bestätigen sich die bekannten Befunde, wonach Schülerinnen und Schüler mit einem Migrationshintergrund des zweiten Migrationsclusters auch unter Kontrolle der primären und sozialen Herkunftseffekte beim Übertritt in die nachobligatorischen Ausbildungen benachteiligt zu sein scheinen. Der positive Effekt fremdsprachiger Schülerinnen und Schüler für den nachobligatorischen Übertritt in die Allgemeinbildung reiht sich ein in eine Reihe von Befunden, welche die These des Immigrant Optimism stützen (Fernãndez-Reino 2016; Tjaden und Hunkler 2017). In Bezug auf Ausbildungslosigkeit kann jedoch genau hier ein Problem vorliegen, nämlich dann, wenn anspruchsvollere nachobligatorische Ausbildungen nicht abgeschlossen werden können und somit wiederum das Risiko für Zertifikatsarmut steigt.

Inwieweit die hier gezeigten Risiken bestimmte Herkunftsgruppen stärker betreffen als andere, und ob sie über den Zeitverlauf bestehen bleiben, also genannte Risikogruppen auch langfristig von Zertifikatsarmut betroffen sind, welche Rolle die Schule und die Lehrpersonen bei der Kompensation dieser Risiken spielen (können), und ob alternative Bildungswege im Schweizerischen Bildungssystem dazu beitragen können, Zertifikatsarmut zu verringern, wäre Gegenstand weitergehender Untersuchungen.

Grundsätzlich kann festgehalten werden, dass ein nicht geringer Teil des Zertifikatsarmutsrisikos, zumindest in der Schweiz, offensichtlich an Kompetenzarmut gekoppelt ist, wie die starken Einflüsse der primären Herkunftseffekte zeigen. Da die momentane Befundlage dafürspricht, dass dies Schülerinnen und Schüler mit und ohne Migrationshintergrund gleichermaßen betrifft, so scheint ein weiterer Ausbau des Angebots an frühkindlicher Bildung insbesondere für weniger wohlhabende bzw. gebildete Familien ein naheliegender und vielversprechender Lösungsansatz.

\section{Literatur}

Allmendinger, J. (1989): Educational systems and labor market outcomes. European Sociological Review, 5. 3, 231-250.

Arrow, K. J. (1973): Higher Education as a Filter. Journal of Public Economics, 2. 3, 193-216. Arrow, K. J. (1985): Collected Papers of Kenneth J. Arrow, Volume 6: Applied Economics. Harvard: Harvard University Press.

Bauer, P. C./Riphahn, R. T. (2010): Kindergartenbesuch und intergenerationale Bildungsmobilität. Vierteljahrshefte zur Wirtschaftsforschung, 79. 3, 121-132.

Beck, M. (2015): Bildungserfolg von Migranten. Bern: Haupt.

Beck, M./Edelmann, D. (2016): Migrationshintergrund und Gender: Eine Überprüfung der statistischen Konstruktion von Differenz am Beispiel der Lehrerinnen- und Lehrerbildung in der Schweiz. In: Kriesi, I./Liebig, B./Horwarth, I./Riegraf, B. (Hrsg.): Gender und Migration in der tertiären Hochschulbildung. Münster: Westfälisches Dampfboot, 168-192. 
Beck, M./Jäpel, F./Becker, R. (2010): Determinanten des Bildungserfolgs von Migranten. In: Quenzel, G./Hurrelmann, K. (Hrsg.): Bildungsverlierer. Wiesbaden: VS Verlag für Sozialwissenschaften, 313-337.

Becker, B. (2010): Bildungsaspirationen von Migranten. MZES Arbeitspapiere, 137.

Becker, B./Gresch, C. (2016): Bildungsaspirationen in Familien mit Migrationshintergrund. In: Diehl, C./Hunkler, C./Kristen, C. (Hrsg.): Ethnische Ungleichheiten im Bildungsverlauf. Mechanismen, Befunde, Debatten. Wiesbaden: Springer VS, 73-115.

Becker, G.S. (1973): The economics of discrimination. Chicago: University of Chicago Press.

Becker, G. S. (1993): Human Capital. Chicago: University of Chicago Press.

Becker, R./Hecken, A.E. (2008): Warum werden Arbeiterkinder vom Studium an Universitäten abgelenkt? Eine empirische Überprüfung der „Ablenkungsthese“ von Müller und Pollak (2007) und ihrer Erweiterung durch Hillmert und Jacob (2003). Kölner Zeitschrift für Soziologie und Sozialpsychologie, 60. 1, 7-33.

Becker, R./Jäpel, F./Beck, M. (2013): Diskriminierung durch Lehrpersonen oder herkunftsbedingte Nachteile von Migranten im Deutschschweizer Schulsystem? Schweizerische Zeitschrift für Soziologie, 39. 3, 517-549.

Becker, R./Lauterbach, W. (2010): Bildung als Privileg - Ursachen, Mechanismen, Prozesse und Wirkungen. In: Becker, R./Lauterbach, W. (Hrsg.): Bildung als Privileg. Wiesbaden: VS Verlag für Sozialwissenschaften, 11-49.

Becker, R./Zangger, C. (2013): Die Bildungsexpansion in der Schweiz und ihre Folgen. Kölner Zeitschrift für Soziologie und Sozialpsychologie, 65. 3, 423-449.

Best, H./Wolf, C. (2012): Modellvergleich und Ergebnisinterpretation in Logit- und Probit-Regressionen. Kölner Zeitschrift für Soziologie und Sozialpsychologie, 64. 2, 377395.

BFS - Bundesamt für Statistik (2017a): Ausbildungsstufen der ständigen Wohnbevölkerung nach Arbeitsmarktstatus, Geschlecht, Nationalität, Altersgruppen und Familientyp. Verfügbar unter: https://www.bfs.admin.ch/bfs/de/home.assetdetail.3783272. html. Zugegriffen: November 2017.

BFS - Bundesamt für Statistik (2017b): Selektion auf der Sekundarstufe I. Verfügbar unter: https://www.bfs.admin.ch/bfs/de/home/statistiken/bildung-wissenschaft/bil dungsindikatoren/bildungssystem-schweiz/themen/bildungsverlaeufe/selektion-se kundarstufe.assetdetail.2861872.html. Zugegriffen: November 2017.

BFS - Bundesamt für Statistik (2017c): Ständige Wohnbevölkerung ab 15 Jahren nach Migrationsstatus und verschiedenen soziodemografischen Merkmalen. Verfügbar unter: https://www.bfs.admin.ch/bfs/de/home/statistiken/kataloge-datenbanken/tabellen. assetdetail.3562525.html. Zugegriffen: November 2017.

BFS - Bundesamt für Statistik (2017d): Übergang in die Sekundarstufe II. Verfügbar unter: https://www.bfs.admin.ch/bfs/de/home/statistiken/bildung-wissenschaft/bil dungsindikatoren/bildungssystem-schweiz/bildungsstufen/sekundarstufe-ii/ueber gang-sekundarstufe2.html. Zugegriffen: November 2017. 
Böni, E./Salm, E. (2008): Multikulturalität in Kindergarten, Primarschule und Sekundarstufe I des Kantons Bern. Schulstatistische Auswertungen mit Daten des Schuljahres 2006. Bern, Erziehungsdirektion des Kantons Bern, Bilungsplanung und Evaluation.

Boudon, R. (1974): Education, Opportunity, and Social Inequality. New York: Wiley.

Bourdieu, P. (1983): Ökonomisches Kapital, kulturelles Kapital, soziales Kapital. In: Kreckel, R. (Hrsg.): Soziale Ungleichheiten. Soziale Welt, Sonderband 2. Göttingen: Schwartz, 183-198.

Breen, R./Goldthorpe, J.H. (1997): Explaining Educational Differentials. Towards a Rational Action Theory. Rationality and Society, 9. 3, 275-305.

Buchmann, C./Dalton, B. (2002): Interpersonal Influences and Educational Aspirations in 12 Countries: The Importance of Institutional Context. Sociology of Education, 75. 2, 99-122.

Coleman, J. S. (1990): Foundations of Social Theory. Cambridge: Harvard University Press. Combet, B. (2013): Zum Einfluss von primären und sekundären Effekten der sozialen Herkunft beim zweiten schulischen Übergang in der Schweiz. Ein Vergleich unterschiedlicher Dekompositions- und Operationalisierungsmethoden. Schweizerische Zeitschrift für Bildungswissenschaften, 35. 3, 447-471.

Diefenbach, H. (2007): Kinder und Jugendliche aus Migrantenfamilien im deutschen Bildungssystem. Wiesbaden, VS Verlag für Sozialwissenschaften.

Diehl, C./Fick, P. (2016): Ethnische Diskriminierung im deutschen Bildungssystem. In: Diehl, C./Hunkler, C./Kristen, C. (Hrsg.): Ethnische Ungleichheiten im Bildungsverlauf. Mechanismen, Befunde, Debatten. Wiesbaden: Springer VS, 243-286.

Ditton, H. (2007): Der Beitrag von Schule und Lehrern zur Reproduktion von Bildungsungleichheit. In: Becker, R./Lauterbach, W. (Hrsg.): Bildung als Privileg. Erklärungen und Befunde zu den Ursachen der Bildungsungleichheit. Wiesbaden: VS Verlag für Sozialwissenschaften, 243-271.

Dollmann, J. (2010): Türkischstämmige Kinder am ersten Bildungsübergang. Wiesbaden: VS.

Dollmann, J. (2016): Der Übergang von der Primar- in die Sekundarstufe. In: Diehl, C./ Hunkler, C./Kristen, C. (Hrsg.): Ethnische Ungleichheiten im Bildungsverlauf. Mechanismen, Befunde, Debatten. Wiesbaden: Springer VS, 517-542.

EDK - Schweizerische Konferenz der kantonalen Erziehungsdirektoren (2017): Das Bildungssystem Schweiz. Verfügbar unter: http://www.edk.ch/dyn/14861.php. Zugegriffen: November 2017.

Erikson, R./Goldthorpe, J.H./Portocarero, L. (1979): Intergenerational class mobility in three Western European societies: England, France and Sweden. The British Journal of Sociology, 30. 4, 415-441.

Esser, H. (2006): Sprache und Integration. Frankfurt New York: Campus.

Fernãndez-Reino, M. (2016): Immigrant optimism or anticipated discrimination? Explaining the first educational transition of ethnic minorities in England. Research in Social Stratification and Mobility, 46. Part B, 141-156. 
Fibbi, R./Lerch, M./Wanner, P. (2006): Unemployment and Discrimination against Youth of Immigrant Origin in Switzerland: When the Name Makes the Difference. Journal of International Migration and Integration, 7. 3, 351-366.

Fritschi, T./Jann, B. (2009): Zum Einfluss vorschulischer Kinderbetreuung auf den Bildungsweg und den erwarteten Erfolg am Arbeitsmarkt. Empirische Pädagogik, 23. 4, 500-520.

Glauser, D. (2015): Berufsausbildung oder Allgemeinbildung. Wiesbaden: Springer VS.

Glauser, D. (2018): Same same but different. Migrationsspezifische Ungleichheiten beim Übergang in Ausbildungen der Sekundarstufe II in der Deutschschweiz. In: Engelage, S. (Hrsg.): Migration und Berufsbildung in der Schweiz. Zürich: Seismo (im Erscheinen).

Gomolla, M./Radtke, F.-O. (2000): Mechanismen institutioneller Diskriminierung in der Schule. In: Gogolin, I./Nauck, B. (Hrsg.): Migration, gesellschaftliche Differenzierung und Bildung. Opladen: Leske + Budrich, 321-341.

Gresch, C. (2012): Der Übergang in die Sekundarstufe I. Wiesbaden: Springer VS.

Gresch, C./Kristen, C. (2011): Staatsbürgerschaft oder Migrationshintergrund? Ein Vergleich unterschiedlicher Operationalisierungsweisen am Beispiel der Bildungsbeteiligung. Zeitschrift für Soziologie, 40. 3, 208-227.

Griga, D. (2014): Participation in Higher Education of Youths with a Migrant Background in Switzerland. Swiss Journal of Sociology, 40. 3, 379-400.

Hadjar, A./Berger, J. (2010): Dauerhafte Bildungsungleichheiten in Westdeutschland, Ostdeutschland und der Schweiz. Eine Kohortenbetrachtung der Ungleichheitsdimensionen soziale Herkunft und Geschlecht. Zeitschrift für Soziologie, 39. 3, 182-201.

Haeberlin, U./Imdorf, C./Kronig, W. (2004a): Chancenungleichheit bei der Lehrstellensuche. Bern und Aarau: Leitungsgruppe des NFP 43. Verfügbar unter: http://www. snf.ch/sitecollectiondocuments/nfp/nfp43_haeberlin_synthesis7.pdf.ZZugegriffen: November 2017.

Haeberlin, U./Imdorf, C./Kronig, W. (2004b): Von der Schule in die Berufslehre. Bern: Haupt.

Heath, A./Brinbaum, Y. (2007): Explaining Ethnic Inequalities in Educational Attainment. Ethnicities, 7. 3, 291-305.

Hoffmann-Nowotny, H.-J. (1973): Soziologie des Fremdarbeiterproblems. Stuttgart: Ferdinand Enke.

Hunkler, C. (2014): Ethnische Ungleichheit beim Zugang zu Ausbildungsplätzen im dualen System. Wiesbaden: Springer VS.

Hunkler, C. (2016): Ethnische Unterschiede beim Zugang zu beruflicher Ausbildung. In: Diehl, C./Hunkler, C./Kristen, C. (Hrsg.): Ethnische Ungleichheiten im Bildungsverlauf. Mechanismen, Befunde, Debatten. Wiesbaden: Springer VS, 597-641.

Hupka, S. (2003): Ausbildungssituationen und -verläufe: Übersicht. In: Bildungsmonitoring Schweiz (Hrsg.): Wege in die nachobligatorische Ausbildung. Die ersten zwei Jahre nach Austritt aus der obligatorischen Schule - Zwischenergebnisse des Jugendlängsschnitts TREE. Neuchâtel: Bundesamt für Statistik (BFS), 33-58. 
Hupka, S./Sachhi, S./Stalder, B.E. (2006): Herkunft oder Leistung? Analyse des Eintritts in eine zertifizierende Nachobligatorische Ausbildung anhand der Daten des Jugendlängsschnitts TREE. Bern, TREE.

Integrationsförderung Stadt Zürich (2008): Ethnic Business. Grundlagen, Hintergründe und Perspektiven. Zürich: Stadt Zürich.

Jann, B./Combet, B. (2012): Zur Entwicklung der intergenerationalen Mobilität in der Schweiz. Schweizerische Zeitschrift für Soziologie, 38. 2, 177-199.

Jäpel, F. (2017): Die Berufsmaturität als Ausbildungsalternative. Bern: Haupt.

Jasso, G. (2007): A New Unified Theory of Sociobehavioral Forces. IZA Discussion Papers, 3243.

Kalter, F. (2008): Ethnische Ungleichheit auf dem Arbeitsmarkt. In: Abraham, M./Hinz, T. (Hrsg.): Arbeitsmarktsoziologie: Probleme, Theorien, empirische Befunde. Wiesbaden: VS, 303-332.

Kao, G./Tienda, M. (1995): Optimism and Achievement: The Educational Performance of Immigrant Youth. Social Science Quarterly, 76. 1, 1-19.

Kappus, E. (2015): Equity: Begriffsbestimmung und Grundsatzfragen für Schule und Bildung. In: EDK (Hrsg.): Equity - Diskriminierung und Chancengerechtigkeit im Bildungswesen. Bern: Schweizerische Konferenz der Eidgenössischen Erziehungsdirektoren (EDK), 9-26.

Kemper, T. (2010): Migrationshintergrund - eine Frage der Definition! Die Deutsche Schule, 102. 4, 315-326.

Kempert, S./Edele, A./Rauch, D. P./Wolf, K. M./Paetsch, J./Darsow, A./Maluch, J./Stanat, P. (2016): Die Rolle der Sprache für zuwanderungsbezogene Ungleichheiten im Bildungserfolg. In: Diehl, C./Hunkler, C./Kristen, C. (Hrsg.): Ethnische Ungleichheiten im Bildungsverlauf. Mechanismen, Befunde, Debatten. Wiesbaden: Springer VS, 157241.

Konsortium PISA.CH (2010): PISA 2009: Schülerinnen und Schüler der Schweiz im internationalen Vergleich. Erste Ergebnisse. Bern und Neuchâtel: BBT/EDK und Konsortium PISA.ch.

Kristen, C. (1999): Bildungsentscheidungen und Bildungsungleichheit - ein Überblick über den Forschungsstand. MZES Arbeitspapiere. 5.

Kristen, C. (2006): Ethnische Diskriminierung in der Grundschule? Die Vergabe von Noten und Bildungsempfehlungen. Kölner Zeitschrift für Soziologie und Sozialpsychologie, 58. $1,79-97$.

Kristen, C./Dollmann, J. (2010): Sekundäre Effekte der ethnischen Herkunft: Kinder aus türkischen Familien am ersten Bildungsübergang. In: Becker, B./Reimer, D. (Hrsg.): Vom Kindergarten bis zur Hochschule. Wiesbaden: VS Verlag für Sozialwissenschaften, $117-144$.

Kronig, W. (2003): Das Konstrukt des leistungsschwachen Immigrantenkindes. Zeitschrift für Erziehungswissenschaft, 6. 1, 126-141.

Kronig, W. (2007): Die systematische Zufälligkeit des Bildungserfolgs. Bern: Haupt. 
Kronig, W./Haeberlin, U./Eckhart, M. (2000): Immigrantenkinder und Schulische Selektion. Bern: Haupt.

Lindenberg, S. (1984): Normen und die Allokation sozialer Wertschätzung. In: Todt, H. (Hrsg.): Normengeleitetes Verhalten in den Sozialwissenschaften. Schriften des Vereins für Socialpolitik. Neue Folge Band 141. Berlin: Duncker \& Humblot, 169-191.

Lockwood, D. (1964): Social Integration and System Integration. In: Zollschan, G. K./ Hirsch, W. (Hrsg.): Explorations in Social Change. New York: Houghton Mifflin, 244257.

Long, S. J. (1997): Regression Models for Categorical and Limited Dependent Variables. Thousand Oaks, CA.: Sage Publications.

Long, S. J./Freese, J. (2014): Regression Models for Categorical Dependent Variables Using Stata. College Station: Stata Press.

Maaz, K./Hausen, C./McElvany, N./Baumert, J. (2006): Stichwort: Übergänge im Bildungssystem. Zeitschrift für Erziehungswissenschaft, 9. 3, 299-327.

Meyer, T. (2003a): Jugendliche mit Migrationshintergrund. In: Bildungsmonitoring Schweiz (Hrsg.): Wege in die nachobligatorische Ausbildung. Die ersten zwei Jahre nach Austritt aus der obligatorischen Schule - Zwischenergebnisse des Jugendlängsschnitts TREE. Neuchâtel: Bundesamt für Statistik (BFS), 111-118.

Meyer, T. (2003b): Ungebildet in die Wissensgesellschaft? Risiken junger Migrantinnen und Migranten auf dem Weg zu einer Berufsqualifikation. terra cognita. 3, 24-29.

Meyer, T./Stalder, B.E./Matter, M. (2003): Bildungswunsch und Wirklichkeit: Thematischer Bericht der Erhebung PISA 2000. Neuchâtel: Bundesamt für Statistik (BFS).

Moser, U. (2001): Der Mythos der Chancengleichheit. Je höher der Bildungsabschluss der Eltern, umso besser die Leistung der Schülerinnen und Schüler - eine empirische Analyse aus dem Kanton Zürich. In: Aeberli, C./Landert, C. (Hrsg.): Potenzial Primarschule. Eine Auslegeordnung, einige weiterführende Ideen und ein Nachgedanke. Zürich: Avenir Suisse,

Moser, U. (2002): Kulturelle Vielfalt in der Schule: Herausforderung und Chance. In: Bildungsmonitoring Schweiz (Hrsg.): Für das Leben gerüstet? Die Grundkompetenzen der Jugendlichen. Bern und Neuenburg: Schweizerische Konferenz der kantonalen Erziehungsdirektoren und Bundesamt für Statistik. Neuchâtel: Bundesamt für Statistik (BFS) und Schweizerische Konferenz der kantonalen Erziehungsdirektoren (EDK) und Bundesamt für Statistik (BFS), 113-135.

Moser, U./Angelone, D./Hollenweger, J./Buff, A. (2011): Nach sechs Jahren Primarschule. Deutsch, Mathematik und motivational-emotionales Befinden am Ende der 6. Klasse. Zürich: Bildungsdirektion Kanton Zürich.

Moser, U./Keller, F./Zimmermann, P. (2008): Soziale Ungleichheit und Fachleistungen. In: Moser, U./Hollenweger, J. (Hrsg.): Drei Jahre danach: Lesen, Wortschatz, Mathematik und soziale Kompetenzen am Ende der dritten Klasse. Oberentfelden: Sauerländer, 115-150. 
Moser, U./Oostlander, J./Tomasik, M.J. (2017): Soziale Ungleichheiten im Leistungszuwachs und bei Bildungsübergängen. In: Neuenschwander, M. P./Nägele, C. (Hrsg.): Bildungsverläufe von der Einschulung bis in den ersten Arbeitsmarkt. Theoretische Ansätze, empirische Befunde und Beispiele. Wiesbaden: Springer VS, 59-77.

Moser, U./Ramseier, E./Keller, C./Huber, M. (1997): Schule auf dem Prüfstand. Eine Evaluation der Sekundarstufe I auf der Grundlage der „Third International Mathematics and Science Study“. Chur Zürich: Rüegger.

Moser, U./Rhyn, H. (2000): Lernerfolg in der Primarschule. Aarau: Sauerländer.

Neuenschwander, M. P. (2012): Selektionsprozesse beim Übergang in die Sekundarstufe I, die Berufsausbildung und die tertiäre Ausbildung. In: Bergman, M. M./Hupka-Brunner, S./Meyer, T./Samuel, R. (Hrsg.): Bildung - Arbeit - Erwachsenwerden. Wiesbaden: Springer VS, 181-201.

Olczyk, M./Seuring, J./Will, G./Zinn, S. (2016): Migranten und ihre Nachkommen im deutschen Bildungssystem: Ein aktueller Überblick. In: Diehl, C./Hunkler, C./Kristen, C. (Hrsg.): Ethnische Ungleichheiten im Bildungsverlauf. Mechanismen, Befunde, Debatten. Wiesbaden: Springer VS, 33-70.

Piguet, E. (2006): Einwanderungsland Schweiz: fünf Jahrzehnte halb geöffnete Grenzen. Bern: Haupt.

Ramseier, E./Brühwiler, C. (2003): Herkunft, Leistung und Bildungschancen im gegliederten Bildungssystem: Vertiefte PISA-Analyse unter Einbezug der kognitiven Grundfähigkeiten. Schweizerische Zeitschrift für Bildungswissenschaften, 25. 1, 23-56.

Rüesch, P. (1998): Spielt die Schule eine Rolle? Bern: Peter Lang.

Schallock, T. (2016): Tracking. Konsequenzen der Leistungsgruppierung von Schülerinnen und Schülern für die Leistungsentwicklung. In: Diehl, C./Hunkler, C./Kristen, C. (Hrsg.): Ethnische Ungleichheiten im Bildungsverlauf. Mechanismen, Befunde, Debatten. Wiesbaden: Springer VS, 287-330.

Schindler, S. (2012): Aufstiegsangst? Eine Studie zur sozialen Ungleichheit beim Hochschulzugang im historischen Zeitverlauf. Düsseldorf: Vodafone Stiftung Deutschland.

Schumann, S. (2011): Leistungs- und Herkunftseffekte beim Hochschulzugang in der Schweiz. Ein Vergleich zwischen Absolventinnen und Absolventen mit gymnasialer Maturität und mit Berufsmaturität. Zeitschrift für Pädagogik, 57. 2, 246-268.

Seibert, H./Solga, H. (2005): Gleiche Chancen dank einer abgeschlossenen Ausbildung? Zum Signalwert von Ausbildungsabschlüssen bei ausländischen und deutschen jungen Erwachsenen. Zeitschrift für Soziologie, 34. 5, 364-382.

Shavit, Y./Muller, W. (200o): Vocational secondary education. European societies, 2. 1, 29-50.

SKBF - Schweizerische Koordinationsstelle für Bildungsforschung (2007): Bildungsbericht Schweiz: 2006. Aarau: Schweizerische Koordinationsstelle für Bildungsforschung (SKBF/CSRE).

SKBF - Schweizerische Koordinationsstelle für Bildungsforschung (SKBF) (2010): Bildungsbericht Schweiz: 2010. Aarau: Schweizerische Koordinationsstelle für Bildungsforschung (SKBF/CSRE). 
SKBF - Schweizerische Koordinationsstelle für Bildungsforschung (2014): Bildungsbericht Schweiz 2014. Aarau: Schweizerische Koordinationsstelle für Bildungsforschung (SKBF).

Söhn, J. (2011): Rechtsstatus und Bildungschancen. Die staatliche Ungleichbehandlung von Migrantengruppen und ihre Konsequenzen. Wiesbaden: VS Verlag für Sozialwissenschaften.

Spence, M. (1973): Job market signaling. The quarterly journal of Economics, 87. 3, 355-374. Stadelmann-Steffen, I. (2011): Education Policy and Educational Inequality - Evidence from the Swiss Laboratory. European Sociological Review, 28. 3, 379-393.

Stamm, M./Kost, J./Suter, P./Holzinger-Neulinger, M./Safi, N./Stroezel, H. (2011): Dropout $\mathrm{CH}$ - Schulabbruch und Absentismus in der Schweiz. Zeitschrift für Pädagogik, 57. 2, 187-202.

Stamm, M./Reinwand, V./Burger, K./Schmid, K./Viehhauser, M./Muheim, V. (2009): Frühkindliche Bildung in der Schweiz. Fribourg: Universität Fribourg.

Stocké, V. (2007): Explaining Educational Decision and Effects of Families' Social Class Position: An Empirical Test of the Breen Goldthorpe Model of Educational Attainment. European Sociological Review, 23. 4, 505-519.

Tjaden, J.D./Hunkler, C. (2017): The optimism trap: Migrants educational choices in stratified education systems. Social Science Research, 67. 213-228.

Tjaden, J. D./Scharenberg, K. (2016): Ethnic choice effects at the transition into upper-secondary education in Switzerland. Acta Sociologica, 60. 4, 309-324.

Trautwein, U./Baeriswyl, F. (2007): Wenn leistungsstarke Klassenkameraden ein Nachteil sind. Zeitschrift für Pädagogische Psychologie, 21. 2, 119-133.

TREE (2016): Dokumentation zur 1. TREE-Kohorte (TREE1), 200o-2016. Bern: TREE.

Van De Werfhorst, H. G./Van Tubergen, F. (2007): Ethnicity, schooling, and merit in the Netherlands. Ethnicities, 7. 3, 416-444.

White, I. R./Royston, P./Wood, A.M. (2011): Multiple imputation using chained equations: Issues and guidance for practice. Statistics in Medicine, 30. 4, 377-399.

Open Access Dieses Kapitel wird unter der Creative Commons Namensnennung 4.0 International Lizenz (http://creativecommons.org/licenses/by/4.0/deed.de) veröffentlicht, welche die Nutzung, Vervielfältigung, Bearbeitung, Verbreitung und Wiedergabe in jeglichem Medium und Format erlaubt, sofern Sie den/die ursprünglichen Autor(en) und die Quelle ordnungsgemäß nennen, einen Link zur Creative Commons Lizenz beifügen und angeben, ob Änderungen vorgenommen wurden. Die in diesem Kapitel enthaltenen Bilder und sonstiges Drittmaterial unterliegen ebenfalls der genannten Creative Commons Lizenz, sofern sich aus der Abbildungslegende nichts anderes ergibt. Sofern das betreffende Material nicht unter der genannten Creative Commons Lizenz steht und die betreffende Handlung nicht nach gesetzlichen Vorschriften erlaubt ist, ist für die oben aufgeführten Weiterverwendungen des Materials die Einwilligung des jeweiligen Rechteinhabers einzuholen. 